\title{
The Delta Scuti star 38 Eri from the ground and from space $^{\star} \dagger$
}

\author{
M. Paparó, ${ }^{1} \ddagger$ Z. Kolláth, ${ }^{2}$ R. R. Shobbrook, ${ }^{3} \S$ J. M. Matthews, ${ }^{4}$ V. Antoci, ${ }^{5}$ \\ J. M. Benkő, ${ }^{1}$ N.-K. Park, ${ }^{6,7}$ M. T. Mirtorabi,${ }^{8}$ K. Luedeke, ${ }^{9}$ A. Kusakin,,${ }^{10}$ \\ Zs. Bognár, ${ }^{1}$ Á. Sódor, ${ }^{1}$ A. García-Hernández, ${ }^{11}$ J. H. Peña, ${ }^{12}$ R. Kuschnig, ${ }^{13,14}$ \\ A. F. J. Moffat ${ }^{15}$ J. Rowe, ${ }^{16}$ S. M. Rucinski, ${ }^{17}$ D. Sasselov, ${ }^{18}$ and W. W. Weiss ${ }^{13}$ \\ Affiliations are listed at the end of the paper
}

Accepted 2018 April 3. Received 2018 March 26; in original form 2017 November 2

\begin{abstract}
We present and discuss the pulsational characteristics of the Delta Scuti star 38 Eri from photometric data obtained at two widely spaced epochs, partly from the ground (1998) and partly from space (MOST, 2011). We found 18 frequencies resolving the discrepancy among the previously published frequencies. Some of the frequencies appeared with different relative amplitudes at two epochs, however, we carried out investigation for amplitude variability for only the MOST data. Amplitude variability was found for one of three frequencies that satisfy the necessary frequency criteria for linear-combination or resonant-mode coupling. Checking the criteria of beating and resonant-mode coupling we excluded them as possible reason for amplitude variability. The two recently developed methods of rotational-splitting and sequence-search were applied to find regular spacings based only on frequencies. Doublets or incomplete multiplets with $l=1,2$ and 3 were found in the rotational splitting search. In the sequence search method we identified four sequences. The averaged spacing, probably a combination of the large separation and the rotational frequency, is $1.724 \pm 0.092 \mathrm{~d}^{-1}$. Using the spacing and the scaling relation $\bar{\rho}=[0.0394,0.0554] \mathrm{gcm}^{-3}$ was derived. The shift of the sequences proved to be the integer multiple of the rotational splitting spacing. Using the precise MOST frequencies and multi-colour photometry in a hybrid way, we identified four modes with $l=1$, two modes with $l=2$, two modes with $l=3$, and two modes as $l=0$ radial modes.
\end{abstract}

Key words: Stars: variables: Delta Scuti - stars: oscillations - stars: interiors - stars: individual: 38 Eri - techniques: photometric - space vehicles

\section{INTRODUCTION}

Delta Scuti stars are located at the intersection of the classical instability strip and the main sequence on the Hertzsprung-Russell diagram (HRD). This region represents

\footnotetext{
* The ground-based observations were obtained at SAAO, South Africa; UNAM San Pedro Martír, Mexico; Siding Spring, Australia; Zahran, Iran; Sobaeksan, Korea, Albuquerque, USA; Almaty, Kazakhstan and La Palma, Spain.

† Based on data from the MOST satellite, a Canadian Space Agency mission, jointly operated by Dynacon Inc., the University of Toronto Institute for Aerospace Studies and the University of British Columbia, with the assistance of the University of Vienna. $\ddagger$ E-mail: paparo@konkoly.hu

$\S$ Dedicated to the memory of R. R. Shobbrook who passed away during the preparation of the paper.
}

a transition zone from radiative cores and thick convective envelopes (low mass stars) to large convective cores and thin convective envelopes (high mass stars). Evolved $\delta$ Scuti stars, which are beyond the main sequence, experience large changes in their interiors in a relatively short period of time after the hydrogen in their core is exhausted. The pulsation modes exhibit mixed characters, a g-mode character near the core and a p-mode character near the surface (Osaki 1975). The complexity of both the radial and non-radial modes excited in these stars and their critical evolutionary stage makes these stars observationally interesting and theoretically challenging. The additional effect of generally being considered moderate and fast rotators (Breger 2000a) increases the difficulty for unique mode identification. We present here the $\delta$ Scuti star 38 Eri, that has proven chal- 
Table 1. Physical parameters of 38 Eri derived by Balona (2000) and McDonald et al. (2017). Both set of parameters were used to calculate the period ratios and pulsation constant of some frequencies.

\begin{tabular}{lll}
\hline & Balona $(2000)$ & McDonald et al. (2017) \\
$\log T_{\text {eff }}$ & $3.85 \pm 0.01$ & $3.85 \pm 0.01$ \\
$\log g$ & $3.60 \pm 0.06$ & 3.514 \\
$M_{V} \mid M_{\text {bol }}$ & $1.11 \pm 0.09 \mathrm{mag}$ & $1.24 \mathrm{mag}$ \\
$\log L / L_{\odot}$ & $1.50 \pm 0.04$ & $1.4077 \pm 0.0006$ \\
$M / M_{\odot}$ & $2.03 \pm 0.07$ & assumed \\
$R / R_{\odot}$ & $3.7 \pm 0.1$ & 3.422 \\
$v \sin i$ & $98 \mathrm{kms}^{-1}$ & \\
$P_{\text {rot }}$ & $<1.9$ day & $<1.8$ day \\
Parallax & $25.98 \pm 1.04$ mas & $26.80 \pm 0.012$ mas \\
\hline
\end{tabular}

lenging even observationally, due to the serious one-day alias problem.

The light variation of 38 Eri ( $o^{1}$ Eri, HR 1298, HD 26574, F2II-III, $V=4.026 \mathrm{mag}$ ) was discovered in a short-period variability survey for bright stars of spectral type $\mathrm{A}$ and $\mathrm{F}$ in the southern hemisphere by Jørgensen, Johansen \& Olsen (1971). The 12-hour observing run revealed a frequency at $13.227 \mathrm{~d}^{-1}$ and another extremely uncertain one at around $10 \mathrm{~d}^{-1}$. The next observing run Jørgensen \& Nørgaard-Nielsen (1975) corrected the value of the dominant mode to $12.27 \mathrm{~d}^{-1}$ and a new peak appeared at $7.74 \mathrm{~d}^{-1}$. The third photometric trial for determining the frequency content better (Poretti 1989) was a oneweek long run consisting of altogether 40 hours of observations. The highest peak was at $13.38 \mathrm{~d}^{-1}$ and a well-defined structure appeared around $6 \mathrm{~d}^{-1}$ with peaks at $6.94 \mathrm{~d}^{-1}$ and $6.03 \mathrm{~d}^{-1}$. The author argued that the two frequencies were definitely not an alias of each other. Some features were better represented in this interpretation but the fit was still not satisfactory. To obtain a better fit additional terms (10.45, 11.84. 6.74 and $8.17 \mathrm{~d}^{-1}$ frequencies) were involved, however these frequencies were uncertain according to the paper.

Two spectroscopic investigations were devoted to 38 Eri. Yang \& Walker (1986) confirmed the presence of nonradial pulsation. At least four different absorption features were localised moving through the spectral line from the high precision, but short-duration radial velocity measurements. The mean period $11.36 \mathrm{~d}^{-1}$ was suggested to be a high degree mode, possibly $l=m=14$.

Balona (2000) presented 247 high-dispersion echelle spectra for 38 Eri. Due to the single-site observation the solution was also affected by the $\pm 1 \mathrm{~d}^{-1}$ alias problem. Frequencies at 11.9 or $12.9 ; 12.4$ or 13.4 and 16.9 or $17.9 \mathrm{~d}^{-1}$ were reported. Significant frequencies were found at 2.68, 13.26 and $9.47 d^{-1}$. The probable identifications were pro-grade and retrograde modes of $l=7$. Balona (2000) derived the physical parameters of 38 Eri from the dedicated high-dispersion (0.06-0.08 ^) spectroscopy that we presented here in Table 1 . His modelling resulted in a post-main sequence evolutionary stage. Over the years new parameters were published. A lower surface gravity value $(\log g=3.39)$ and a similar effective temperature $\left(\log T_{\text {eff }}=3.84\right)$ were published by Gray et al. (2006). However, the actual resolution was closer to $3.5 \AA$ that is perfect for the original goal, for the classification purpose of a large sample. The newly reduced Hippar$\cos$ measurements revised the parallaxes to $26.80 \pm 0.32$ mas (van Leeuwen 2007). McDonald, Zijlstra \& Watson (2017) based on the Gaia measurements, published the parameters of 38 Eri that we also present in Table 1. According to the paper, the surface gravity was calculated from assumed mass. We calculated the rotation period using their radius and the rotational velocity supposing $i=90^{\circ}$ inclination, similar to Balona (2000). We used both set of values for calculating the pulsation constant and period ratios for some frequencies of 38 Eri.

The present investigation is the next step in resolving the complex pulsational behaviour of 38 Eri.

\section{NEW OBSERVATIONS}

\subsection{Ground-based multi-site campaign in 1998}

The only chance in 1998 was a multi-site campaign to resolve the severe $\pm 1 \mathrm{~d}^{-1}$ alias problem. The campaign extended from mid-October to the end of December in 1998. Three sites were dominant regarding the time and the amount of the data that were devoted to the joint efforts. These three sites gave the compact, best coverage part of the campaign. Some other sites also joined the campaign but with less contribution. However, they extended the time-base of the campaign. Table 2 gives the sites, the telescope size that was used, their time-base, and the number of clear skies during the time span. For better comparison the coverage was counted for each observing site. The last column contains the filter(s) that were used in the measurements.

The following instruments were applied:

- South-African Astronomical Observatory, Sutherland, South Africa: single-channel Modular Photometer with a HAMAMATSU R943-02 Gallium Arsenide tube (Kilkenny et al. 1988) with a neutral density filter.

- UNAM, San Pedro Martír, Mexico: a single-channel photometer with a dry-ice-cooled 1P21 photomultiplier, with a neutral density filter.

- ANU, Siding Spring Observatory, Australia: the 'Monitored Filter Box' photometer with an 8-hole filter wheel and computer controlled rotations was used. A neutral density filter was used to be able to cover both the Strömgren and Johnson passbands.

- Sobaeksan Observatory, Korea: Photometrics PM512+NU200 Liquid Nitrogen Cooled CCD Camera coupled with ADPS (Automatic Differential Photometry System).

- Institute for Advanced Studies in Basic Sciences Observatory (IASBS), Zanjan, Iran: SSP-3 photometer was attached to a $14^{\prime \prime}$ Celestron telescope.

- Albuquerque, USA: an SSP-3 photometer was attached to a 10-inch Meade LX200 telescope

- Almaty, Kazakhstan: an AZT-14 telescope with an AMF-6 photometer was used.

Table 3 lists the journal of the observation with dates on which observations were obtained, the time of the beginning of observations in HJD, the duration of those observations in hours and decimals, the number of measurements of 38 Eri and the name of the observers. Subtotal of the observing time and the number of the measurements are given for each site. The total values for the whole campaign are given in the last line. 
Table 2. List of observing sites and the contribution to the campaign. The best coverage was in South Africa and Mexico. Australian contribution in two photometric systems are highly valuable.

\begin{tabular}{llcccc}
\hline Site & $\begin{array}{l}\text { Telescope } \\
(\mathrm{m})\end{array}$ & $\begin{array}{c}T_{\text {base }} \\
(\mathrm{d})\end{array}$ & $\begin{array}{c}\text { No. nights } \\
(\mathrm{d})\end{array}$ & $\begin{array}{c}\text { Cov. of total run } \\
(\%)\end{array}$ & Filter \\
\hline SAAO, Sutherland, South Africa & 0.5 & 25 & 11 & 26.2 & uvby, I \\
UNAM, San Pedro Martír, Mexico & 0.84 & 15 & 13 & 27.3 & BVI \\
Siding Spring, Australia & 0.6 & 26 & 9 & 6.3 & vby, UBVRI \\
IASBS, Zanjan, Iran & 0.35 & 42 & 6 & 2.4 & $V$ \\
Sobaeksan Observatory, Korea & 0.61 & 48 & 4 & 2.6 & $y$ \\
Albuquerque, USA & 0.25 & 50 & 6 & 0.8 & $V$ \\
Fesenkov Observatory, Almaty, Kazkhstan & 0.48 & & 1 & & $V$ \\
\hline
\end{tabular}

At majority of the sites the C1= HR $1272(\mathrm{~A} 1 \mathrm{~V})$ and $\mathrm{C} 2=\mathrm{HR} 1290$ (G8III) were used as comparison stars. The observing cycle was usually $\mathrm{C} 1, \mathrm{~V}, \mathrm{C} 2, \mathrm{~V}, \mathrm{C} 1, \ldots$ Regarding the brightness of 38 Eri at some sites, using larger telescopes, a neutral density filter was used. The integration time was rather different on the different sites from 2 sec (de-focused $\mathrm{CCD}$ ) to $30-40 \mathrm{sec}$ (neutral filter), due to the different instruments, filters and weather conditions. In order to assign similar weight, the integrations were averaged to get a sampling as dense as at other sites. The sampling times were 6-7 minutes at most sites. Siding Spring observatory has a slightly longer sampling time (7-9 minutes) due to the longer observing cycles using most passbands of two photometric systems (Strömgren and Johnson). This contribution turned out to be highly important in the mode identification.

If the observing run was long enough, the nightly determined extinction coefficients were used. In other cases mean values were applied. If some trend remained it was subtracted as a straight line. The V-C1 (38 Eri - HR 1272) relative light curve was used for the frequency analyses. The C1-C2 curve (HR 1272 - HR 1290) was used for checking the non-variability of the $\mathrm{C} 1=\mathrm{HR} 1272$ comparison star. In a single night only $\mathrm{C} 2=\mathrm{HR} 1290$ was observed. The $\mathrm{V}-\mathrm{C} 1$ curve was determined using the literature value of the $\mathrm{C} 1-\mathrm{C} 2$.

Different photometer/filter combinations lead to different zero points, even if the same comparison stars were used. This fact needs to be considered when different data sets are combined. The differential light curves showed that the zero points of the instrumental system on the different sites were slightly different. All light curves were shifted to the zero point by subtracting the nightly mean value.

Combining the data of each site in a certain colour resulted in the campaign data for 1998. Table 2 shows that both Strömgren vby and Johnson-Cousins BVI measurements were obtained at least at two sites. The observations of R. Shobbrook at Siding Springs were connected partly to the Strömgren observations in South Africa and the Johnson $B V I$ observation in Mexico.

\subsection{MOST data from 2011}

The MOST (Microvariability and Oscillation of STars) Canadian Space Telescope (Walker et al. 2003) was launched on June 30, 2003 into a low-Earth (altitude 820 $\mathrm{km})$, Sun-synchronous circular polar orbit with an orbital period of 101 minutes. The orbital period corresponded to an orbital frequency of $14.2 \mathrm{~d}^{-1}$. The light was collected by
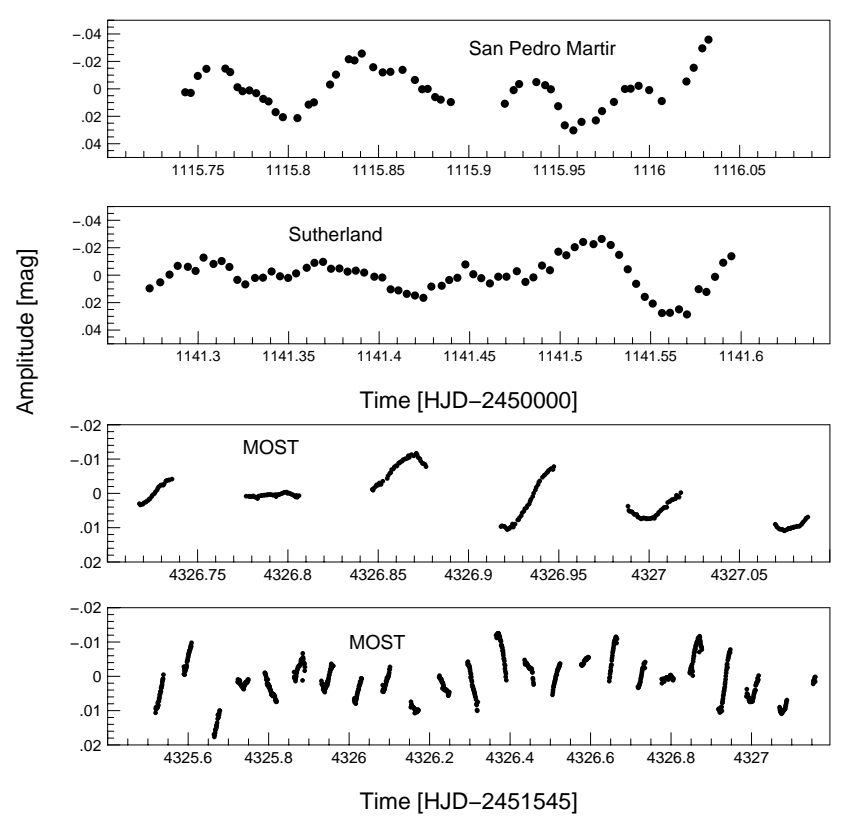

Figure 1. Light curves at San Pedro Martír, South-Africa from ground and by MOST from space. The upper three panels show the quality of the ground-based data and the space photometry on the same scale. The regular gaps in the MOST data are shown in the bottom panel.

a 15-cm aperture Rumak-Maksutov telescope to two frametransferred CCD cameras, through a single, wide passband filter (350-700 nm). One CCD camera was planned only for tracking the telescope, while other was used for scientific purposes. After dramatically reducing the tracking jitter in 2005, the MOST telescope simultaneously supplied three types of photometric data for 8 weeks in the Continuous Viewing Zone (CVZ) of declination range of $+34>\delta>-18$.

In the Fabry imaging mode the bright stars illuminate the CCD through a Fabry jet lens. The Direct Imaging mode resembles conventional CCD photometry where photometry is obtained from slightly defocused images of stars. In the Guide Star Photometry mode the MOST Star tracker (the originally non-scientific CCD camera) was used for photometry. The satellite operations were upgraded after the launch, so the Attitude Control System (ACS) helped in providing more highly accurate photometry (see, e.g. Walker et al. 2005; Aerts et al. 2006).

MOST was designed to achieve the mission's primary goal of detecting rapid photometric oscillations (period of 
Table 3. The journal of observations. The seven sites are ideally distributed in longitude. The total length gives the whole effort independently of colours.

\begin{tabular}{|c|c|c|c|c|c|}
\hline \multirow{2}{*}{$\begin{array}{l}\text { Site } \\
\text { Siding Spring }\end{array}$} & \multirow{2}{*}{$\begin{array}{l}\text { Date } \\
1998 \\
14 \text { Nov }\end{array}$} & \multirow{2}{*}{$\begin{array}{c}\text { HJD- } \\
2450000\end{array}$} & \multirow{2}{*}{$\begin{array}{r}\text { Length } \\
(\mathrm{h})\end{array}$} & \multicolumn{2}{|c|}{$\mathrm{N}$ Observer } \\
\hline & & & & 16 & $\mathrm{RS}$ \\
\hline & $15 \mathrm{Nov}$ & 1133.02 & 1.38 & 10 & $\mathrm{RS}$ \\
\hline & $16 \mathrm{Nov}$ & 1133.95 & 4.83 & 25 & $\mathrm{RS}$ \\
\hline & $20 \mathrm{Nov}$ & 1137.94 & 6.82 & 38 & $\mathrm{RS}$ \\
\hline & $23 \mathrm{Nov}$ & 1140.99 & 4.43 & 17 & $\mathrm{RS}$ \\
\hline & $26 \mathrm{Nov}$ & 1144.05 & 3.75 & 19 & $\mathrm{RS}$ \\
\hline & $29 \mathrm{Nov}$ & 1146.94 & 6.34 & 33 & $\mathrm{RS}$ \\
\hline & $01 \mathrm{Dec}$ & 1148.96 & 6.39 & 33 & $\mathrm{RS}$ \\
\hline & 11 Dec & 1158.07 & 3.17 & 18 & $\mathrm{RS}$ \\
\hline \multicolumn{2}{|l|}{ Subtotal } & & 39.57 & 209 & \\
\hline \multirow[t]{13}{*}{ San Pedro Martír } & 26 Oct & 1113.79 & 5.33 & 22 & ZK/JP \\
\hline & 27 Oct & 1114.79 & 7.11 & 51 & ZK \\
\hline & 28 Oct & 1115.74 & 7.20 & 66 & ZK \\
\hline & 31 Oct & 1118.72 & 7.11 & 74 & ZK \\
\hline & $01 \mathrm{Nov}$ & 1119.72 & 7.38 & 71 & $\mathrm{ZK}$ \\
\hline & $02 \mathrm{Nov}$ & 1120.77 & 6.46 & 68 & ZK \\
\hline & $03 \mathrm{Nov}$ & 1121.71 & 7.33 & 80 & ZK \\
\hline & $04 \mathrm{Nov}$ & 1122.72 & 7.11 & 76 & ZK \\
\hline & $05 \mathrm{Nov}$ & 1123.72 & 7.02 & 52 & ZK \\
\hline & $06 \mathrm{Nov}$ & 1124.73 & 6.99 & 59 & ZK \\
\hline & $07 \mathrm{Nov}$ & 1125.86 & 4.25 & 31 & ZK \\
\hline & $09 \mathrm{Nov}$ & 1127.72 & 7.39 & 70 & ZK \\
\hline & $10 \mathrm{Nov}$ & 1128.72 & 7.38 & 38 & ZK \\
\hline \multicolumn{2}{|l|}{ Subtotal } & & 88.06 & 758 & \\
\hline \multirow[t]{11}{*}{ Sutherland } & $04 \mathrm{Nov}$ & 1122.31 & 7.31 & 36 & MP \\
\hline & $07 \mathrm{Nov}$ & 1125.30 & 7.29 & 60 & MP \\
\hline & $08 \mathrm{Nov}$ & 1126.30 & 7.48 & 62 & MP \\
\hline & $10 \mathrm{Nov}$ & 1128.31 & 7.36 & 46 & MP \\
\hline & $11 \mathrm{Nov}$ & 1129.29 & 7.68 & 58 & MP \\
\hline & $21 \mathrm{Nov}$ & 1139.40 & 4.85 & 43 & MP \\
\hline & $23 \mathrm{Nov}$ & 1141.27 & 5.46 & 70 & MP \\
\hline & $24 \mathrm{Nov}$ & 1142.31 & 5.14 & 45 & MP \\
\hline & $25 \mathrm{Nov}$ & 1143.27 & 4.67 & 43 & MP \\
\hline & $28 \mathrm{Nov}$ & 1146.32 & 6.06 & 54 & MP \\
\hline & $29 \mathrm{Nov}$ & 1147.32 & 5.87 & 53 & MP \\
\hline Subtotal & & & 69.17 & 570 & \\
\hline \multirow[t]{6}{*}{ Iran } & 27 Oct & 1114.34 & 3.85 & 36 & $\mathrm{TM}$ \\
\hline & 31 Oct & 1118.31 & 2.78 & 30 & $\mathrm{TM}$ \\
\hline & $03 \mathrm{Nov}$ & 1121.31 & 5.04 & 52 & TM \\
\hline & $13 \mathrm{Nov}$ & 1130.33 & 3.87 & 42 & $\mathrm{TM}$ \\
\hline & $25 \mathrm{Nov}$ & 1143.26 & 3.89 & 46 & $\mathrm{TM}$ \\
\hline & 08 Dec & 1156.29 & 4.56 & 42 & $\mathrm{TM}$ \\
\hline Subtotal & & & 23.99 & 248 & \\
\hline \multirow[t]{4}{*}{ Korea } & $11 \mathrm{Nov}$ & 1129.13 & 4.32 & 37 & NKP \\
\hline & $28 \mathrm{Nov}$ & 1146.04 & 5.40 & 35 & NKP \\
\hline & 27 Dec & 1174.94 & 5.88 & 51 & NKP \\
\hline & 29 Dec & 1176.95 & 3.00 & 27 & NKP \\
\hline Subtotal & & & 18.6 & 150 & \\
\hline \multirow[t]{6}{*}{ USA } & 19 Oct & 1105.79 & 1.99 & 27 & KL \\
\hline & $05 \mathrm{Nov}$ & 1122.73 & 1.91 & 25 & KL \\
\hline & $14 \mathrm{Nov}$ & 1131.81 & 1.89 & 27 & KL \\
\hline & $22 \mathrm{Nov}$ & 1139.81 & 1.90 & 27 & KL \\
\hline & $24 \mathrm{Nov}$ & 1141.76 & 1.14 & 14 & KL \\
\hline & 08 Dec & 1155.71 & 0.98 & 14 & KL \\
\hline Subtotal & & & 9.81 & 134 & \\
\hline Kazakhstan & 08 Dec & 1156.19 & 3.57 & 23 & AK \\
\hline Total & & & 252.77 & 2092 & \\
\hline
\end{tabular}

several minutes) in bright pulsating stars with a precision of approximately 1 parts per million ( $\mu$ mags) in the Fourier domain. MOST was intended for non-differential photometery, since the relatively high frequencies of the oscillations can be clearly distinguished in the Fourier spectrum of the data from possible drifts and noise (Matthews 2004). However, the MOST space-based photometer could also monitor bright $\delta$ Scuti stars, with slower oscillation than the original goal, with an amplitude detection limit of a few $\mu$ mag. Matthews (2004) predicted (based on a rotational simulation on MOST data) that the rotational splitting of $\delta$ Scuti stars is so complex that even the improved resolution due to the long and continuous time base $(\approx 0.2 \mu \mathrm{Hz}$ equals to $0.01728 \mathrm{~d}^{-1}$ ) and sensitivity (ppms) possible from space observations do not necessarily yield unambiguous result. Since 38 Eri with its $\delta_{2000}=-6^{\circ} 50^{\prime} 16^{\prime \prime}$ position fits the MOST Viewing Zone, we hoped that the MOST observations could help to solve the ambiguities of the frequencies previously published.

The target, 38 Eri was observed by MOST from Nov 05, 2011 to Dec 04, 2011 in the Fabry imaging mode. It shared each MOST orbit with two other Prime Science targets, so there were gaps spaced by the MOST orbital period of about 101 minutes. The individual exposure was 3.0second long, but stacked for a sampling cadence of once per 30 seconds. The modulation of scattered Earth shine at the orbital period of the MOST satellite was filtered by subtracting a linear fit ('sky' versus star) from the data. This method shows the best result and modulates the stellar signal least but gets rid of the orbital period quite nicely. The reduced data presented by the MOST team contains HJD = HJD $_{\text {star }}-$ HJD $_{\text {MOST }}$ where HJD MOST $=51545.0$, the standard zero-point epoch of MOST. In the second column the brightness as mag $=-2.5 \log \left(\mathrm{ADU} \mathrm{pix}^{-1} \mathrm{sec}^{-1}\right)$ is given normalised to the mean. The light curve has the time base of 29.5 days (Rayleigh resolution is $0.034 \mathrm{~d}^{-1}$ ) and 11187 measurements. A sample of the most compact ground-based light curves and two subsets of the MOST light curve are given in Figure 1. The upper three panels show the quality of the ground-based data at San Pedro Martír (Mexico) and Sutherland (South-Africa), and the space photometry by MOST on the same scale. The bottom panel shows the regular gaps in the MOST data over the orbital period. Obviously, the space data are superior to the ground-based observations despite the gaps ${ }^{1}$.

\subsection{Spectroscopy from 2013}

We obtained 69 high-resolution spectra with the HERMES spectrograph at the 1.2-m Mercator Telescope on La Palma (Raskin et al. 2011), Canary Islands, Spain between 14 and 23 January, 2013. The fiber-fed echelle spectrograph was operated in high-resolution (HRF) mode, providing $R=85000$ resolving power. The spectra were reduced with the dedicated reduction pipeline of the HERMES instrument, including bias and stray-light subtraction, echelle-order extraction, flat-field correction, cosmics filtering, and wavelength calibration using ThAr wavelength reference spectra which

1 All photometric data obtained from the ground and from space are given in the electronic tables attached to this paper. 
Table 4. Colour observations. The longest data sets were obtained in Johnson $V$ and Strömgren $y$. The fourth column gives the coverage for the whole data set and in some colours for the most compact part, too.

\begin{tabular}{lrrcc}
\hline Filter & $\begin{array}{r}\text { Length } \\
(\mathrm{h})\end{array}$ & $\mathrm{N}$ & $\begin{array}{c}\text { Coverage } \\
(\%)\end{array}$ & Observer \\
\hline$u$ & 74.70 & 535 & 12.0 & $\mathrm{MP}$ \\
$v$ & 107.20 & 722 & $12.1 / 16.5$ & $\mathrm{MP}, \mathrm{RS}$ \\
$b$ & 113.53 & 803 & 17.5 & $\mathrm{MP}, \mathrm{RS}$ \\
$y$ & 122.82 & 820 & 9.3 & $\mathrm{MP}, \mathrm{RS}, \mathrm{NKP}$ \\
$U$ & 32.90 & 131 & $5.5 / 9.1$ & $\mathrm{RS}$ \\
$B$ & 107.18 & 800 & $9.9 / 12.8$ & $\mathrm{ZK}, \mathrm{RS}$ \\
$V$ & 143.60 & 1030 & $11.7 / 17.1$ & $\mathrm{ZK}, \mathrm{RS}, \mathrm{TM}, \mathrm{KL}, \mathrm{AK}$ \\
$R$ & 29.83 & 131 & $5.0 / 9.1$ & $\mathrm{RS}$ \\
$I$ & 76.50 & 765 & $14.0 / 19.1$ & $\mathrm{MP}, \mathrm{RS}, \mathrm{ZK}$ \\
\hline
\end{tabular}

were obtained several times during the night (Raskin et al. 2011). Afterwards, we normalised the spectra employing an in-house developed automatic tool by Á.S. based on expected continuum wavelength-sections relying on a synthetic F0 stellar spectrum. Finally, we cross-correlated the spectra with a theoretical F0-type spectrum of Solar metallicity without line broadening.

\section{FREQUENCY ANALYSIS}

\subsection{Spectroscopic analysis}

The 69 high-resolution spectra obtained in a one-week long interval were not adequate enough in order to find the frequencies. Nevertheless, we derived the $v \sin i$ and the dominant frequency from the data at hand.

\subsubsection{Projected rotational velocity}

We used the FAmiAs software (Zima 2008b,a) to fit a rotationally broadened absorption-line profile to our mean crosscorrelation function (CCF) in order to derive $v \sin i$ of 38 Eri. The best-fit model has a $v \sin i=101 \mathrm{~km} \mathrm{~s}^{-1}$ projected rotational velocity and $\sigma=10 \mathrm{~km} \mathrm{~s}^{-1}$ intrinsic line-width. The mean CCF along with the fit is plotted in Fig. 2.

Note the strong asymmetry and irregularities of the average CCF. This is most probably, due to the low number (69) of spectroscopic observations which are not enough to average out the complex multi-periodic pulsational variations from the mean profile. Furthermore, the pulsational line-profile variations, as a kind of macro-turbulence, contribute to the line-broadening. Thus, the real $v \sin i$ value might be below the obtained value of $101 \mathrm{~km} \mathrm{~s}^{-1}$. Unfortunately, the low number of spectra does not allow us to model the pulsational line-profile variations; therefore, the precise amount of this contribution is unknown. Practically our solution fits the previously determined $v \sin i$, namely $98 \mathrm{~km} \mathrm{~s}^{-1}$ by Baglin et al. (1973) and Balona (2000).

\subsubsection{Frequency analysis from spectroscopy}

We investigated the line-profile variations in the CCFs by Fourier-analysis using the pixel-by-pixel method, and also

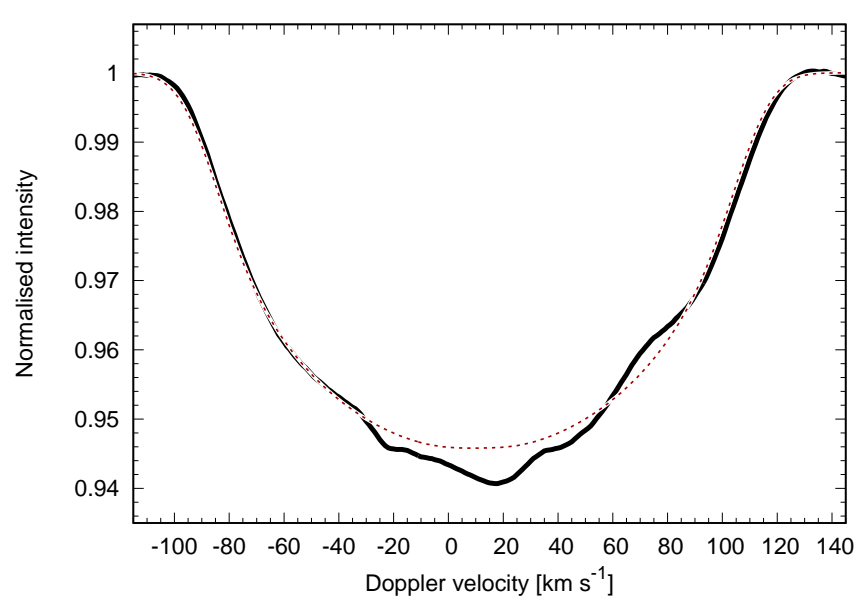

Figure 2. The mean CCF of 38 Eri (thick black line) and the fitted rotationally broadened line profile (thin dashed curve).

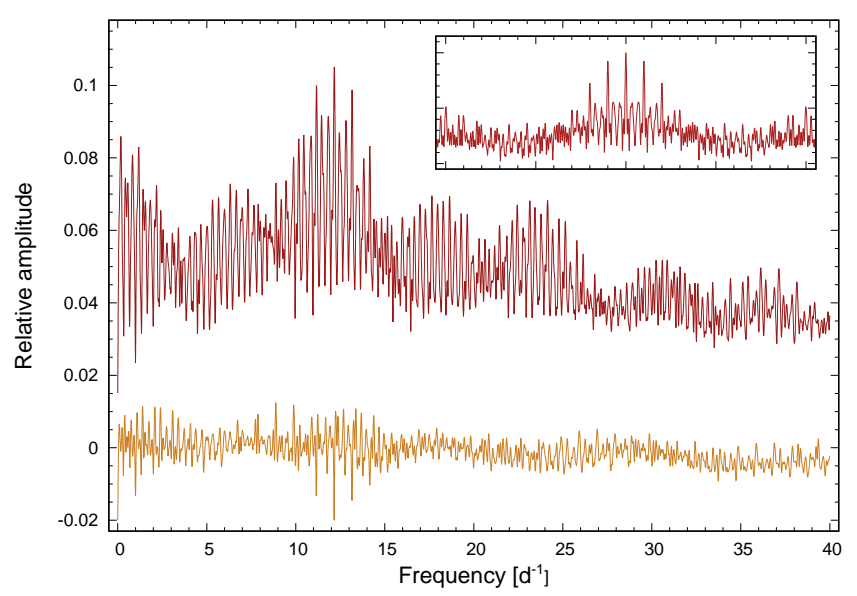

Figure 3. Fourier pixel-by-pixel spectrum of the CCFs of 38 Eri. Top: original spectrum. Bottom: spectrum pre-whitened by the significant variation of $12.16 \mathrm{~d}^{-1}$ (shifted by -0.02 in relative amplitude for better visibility). Insert shows the window function.

with FAmias (Zima 2008b,a). Fig. 3 shows the Fourier spectrum of the CCF variations averaged over the dispersion range -80 to $+110 \mathrm{~km} \mathrm{~s}^{-1}$. Note that the exact choice of the dispersion range does not affect the overall result. The highest peak is at $12.16 \mathrm{~d}^{-1}$. Our observations were affected by daily aliases, as the spectral window function in the insert of Fig. 3 demonstrates. Therefore, the real variation frequency might be $\pm 1 \mathrm{~d}^{-1}$ off from this value. The bottom function in Fig. 3 shows the residual spectrum after the $12.16 \mathrm{~d}^{-1}$ variation had been pre-whitened from the data. Due to the low number of our spectroscopic observations, no further significant line-profile variation frequencies could be identified. 


\subsection{Frequency analyses of photometric data}

The multi-frequency analysis of 38 Eri was performed with the MuFrAn program (Kolláth 1990). MuFrAn (MUlti FRequency ANalysis) is a collection of frequency determination, sine fitting for observational data and graphics routines for the visualisation of the results. At each step all previous frequencies were computed. PERIOD04 was only used for getting the variability of the given frequency. MUFRAN does not have this option. The two programs, tested during the work on the first CoRoT RR Lyrae star (Chadid et al. 2010), are completely equivalent concerning the results on the frequencies.

The periodicity search was carried out for both the ground-based and MOST data. The ground-based campaign provided a differential light curve of $\mathrm{V}-\mathrm{C} 1$, which was analysed for the frequencies. After checking the frequency content of $\mathrm{C} 1-\mathrm{C} 2$ differential light curve we concluded that every peak in the Fourier spectrum of the $\mathrm{V}-\mathrm{C} 1$ differential light curve is attributed to the variability of 38 Eri.

The MOST data present a non-differential light variation of 38 Eri. The peaks of the Fourier spectrum are attributed to the light variation of 38 Eri, although aliases due to the orbital period appear. Peaks in the low-frequency region may appear as an alias pattern caused by the orbital period (linear combination of the excited modes and the orbital period).

Figure 4 contains the Fourier spectra of the groundbased data (in Johnson $V$ and Strömgren $b$ colours) and the MOST data. Despite the 17 percent coverage of the groundbased colour data (that is half of the MOST coverage), the original Fourier spectrum shows that the effect of the $1 \mathrm{~d}^{-1}$ alias in the ground based data was much more severe than the alias of the orbital period in the MOST Fourier spectrum. We expected that the variability of 38 Eri is much better reproduced by the frequencies obtained by MOST. We can also see at first glance from the two panels that the frequency content is rather different in the different passbands. The obviously present peak at around $6.5 \mathrm{~d}^{-1}$ in Johnson $V$ band does not seem to be present/dominant in the Strömgren $b$ band. There were so different solutions in the previous analyses of 38 Eri at different epochs, and in some sense the ground-based campaign and the MOST data also resulted in different solutions that we present both analyses in a bit more detail.

\subsubsection{Frequencies in the ground-based campaign}

The observation in different colours provided the possibility to obtain the frequencies from data of different time-length, thus producing different spectral windows. The resolution of frequencies is lower from the shorter time span. However, the more compact data distribution produces a less complicated spectral window, which means less alias problem. Table 4 gives details on the colour observation: the filter, the observing length in hours and decimals, the number of measurements, the coverage for all and for the compact part, as well as the observers.

We performed the frequency analysis independently for each data set, for different observers and summing up for different colours. The independent analysis meant that the consecutive frequency of the largest amplitude was picked up. We did not have an 'a priori' frequency solution in our mind from the previously published frequencies or from the analyses of the previous data sets. Although the length of the data in different passbands and the Rayleigh resolution were the same $\left(0.028 \mathrm{~d}^{-1}\right)$ the analyses resulted in different sets of frequencies. However, the dominant frequency was definite at around $13.36 \mathrm{~d}^{-1}$ in each passband, something similar to what Poretti (1989) arrived at from photometry and Balona (2000) from spectroscopy. There was a $-1 \mathrm{~d}^{-1}$ alias ambiguity in Balona (2000)'s result.

The consecutive 2-3 steps showed a colour-dependent diversity. We do not present all steps of the frequency search process in each colour here. In Figure 5 we show only some representative Fourier spectra, where we can follow the different frequencies appearing in the second and third steps. The third frequency is labelled by * after the frequency's value in each panel.

In Johnson $V$ the second peak of the highest amplitude is $10.201 \mathrm{~d}^{-1}$, that has never been reported before. We do not force the explanation that it could be the $-2 \mathrm{~d}^{-1}$ alias of $12.27 \mathrm{~d}^{-1}$ reported by Jørgensen \& Nørgaard-Nielsen (1975). The third peak at $6.493 \mathrm{~d}^{-1}$ has not been reported either. Only a well-defined structure around $6 \mathrm{~d}^{-1}$ with a peak at 6.94 and $6.03 \mathrm{~d}^{-1}$ was mentioned by Poretti (1989).

In Strömgren $y$ colour also a new, previously not reported peak appeared at $5.267 \mathrm{~d}^{-1}$ as a second peak. The third peak with nearly the same amplitude was $10.429 \mathrm{~d}^{-1}$, that is close to the $10.45 \mathrm{~d}^{-1}$ uncertain solution given by Poretti (1989). Surprisingly the third peak is almost at twice the value of the second peak. A joint analysis of Johnson $V$ and Strömgren $y$ light curves were also carried out. Beside the dominant peak at $13.36 \mathrm{~d}^{-1}$, the second and third frequencies are $10.201 \mathrm{~d}^{-1}$ and $10.481 \mathrm{~d}^{-1}$ in the frequency search process.

In Strömgren $v$ colour the second peak was at $5.478 \mathrm{~d}^{-1}$ which could be the $-1 \mathrm{~d}^{-1}$ alias of the second peak found in Johnson $V$ colour. However, the third peak at 10.967 is also almost twice the value of the second peak. If we compare the $2.68 \mathrm{~d}^{-1}$ poorly determined frequency given by Balona (2000) to the 5.478 and $10.967 \mathrm{~d}^{-1}$ frequencies, we are reminded of a well-known process of chaotic behaviour, period bifurcation. Of course, the ground-based data are not as high quality for having such a strong conclusion, but it is worthwhile to mention this surprising numerology.

For providing a complete view on how different the frequencies in the different colour bands are, we present the complete frequency list of the ground-based color observations. Table 5 and Table 6 contain the frequencies obtained in the Johnson-Cousins BVI and Strömgren uvby colours, respectively. The frequencies are listed according to decreasing amplitude. Beside the frequencies and amplitudes, the tables show a link to the finally accepted MOST frequencies given later in Table 7, and discussed in Sec 3.2.3.

\subsubsection{Frequencies in MOST}

The diversity of the ground-based results shows that it was worth updating the ground-based campaign data with the MOST observations. The original spectrum in Figure 4 shows how much more definite the frequency content of the MOST data for 38 Eri was. There is no +1 or $-1 \mathrm{~d}^{-1}$ alias and the final frequency list shows why the single-site data 

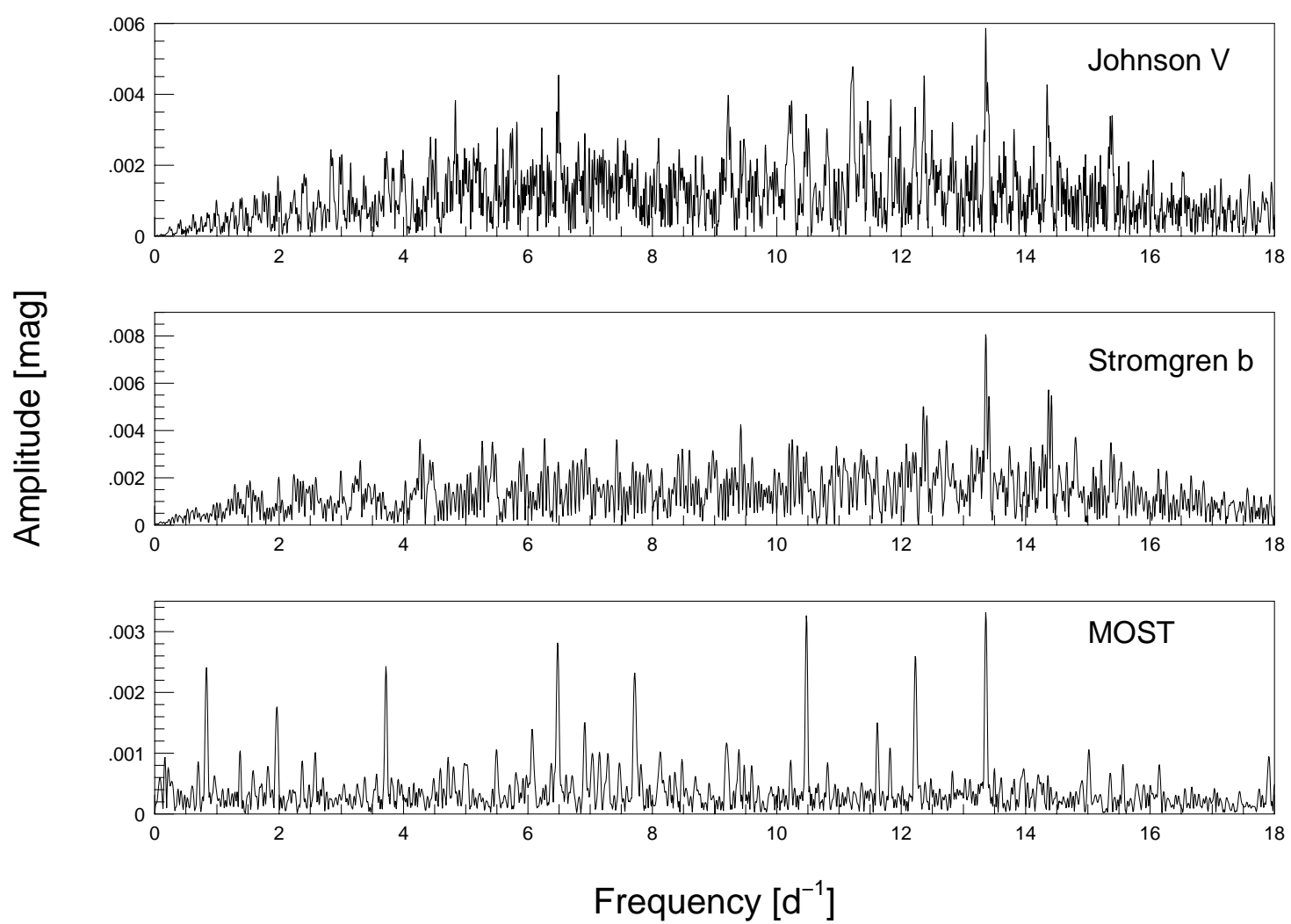

Figure 4. Fourier spectrum in Johnson $V$, Strömgren $b$ colours and MOST data. The first two panels show how different the frequency content is in the different passbands, although the spectral windows are similar (see time base and coverage in Tables 2 and 4 . The MOST spectrum is much cleaner; the peaks are more pronounced due to the higher precision and better data distribution. The peaks below $4 \mathrm{~d}^{-1}$ disappear in the frequency extraction procedure.

Table 5. Frequencies in Johnson-Cousins colours. The frequency solutions were independently obtained. Beside the frequencies and amplitudes the links to the MOST solution are given. The Rayleigh resolution at the ground-based data is $0.028 d^{-1}$.

\begin{tabular}{|c|c|c|c|c|c|c|c|c|}
\hline ID & $\begin{array}{c}\text { Johnson } B \\
\text { Frequency } \\
\left(\mathrm{d}^{-1}\right)\end{array}$ & $\begin{array}{c}\text { Amp } \\
(\mathrm{mmag})\end{array}$ & ID & $\begin{array}{r}\text { Johnson } V \\
\text { Frequency } \\
\left(\mathrm{d}^{-1}\right)\end{array}$ & $\begin{array}{c}\text { Amp } \\
(\mathrm{mmag})\end{array}$ & ID & $\begin{array}{l}\text { Cousins } I \\
\text { Frequency } \\
\qquad\left(\mathrm{d}^{-1}\right)\end{array}$ & $\begin{array}{c}\text { Amp } \\
(\mathrm{mmag})\end{array}$ \\
\hline$f_{1}$ & 13.364 & 9.58 & $f_{1}$ & 13.362 & 6.12 & $f_{1}$ & 13.359 & 4.61 \\
\hline$f_{18}$ & 10.214 & 7.41 & $f_{18}$ & 10.201 & 5.43 & $f_{5}$ & 11.622 & 2.08 \\
\hline$f_{2}$ & 10.481 & 4.99 & $f_{3}$ & 6.493 & 5.46 & $f_{3}+1$ & 7.436 & 2.52 \\
\hline$f_{3}$ & 6.499 & 5.02 & $f_{17}$ & 11.832 & 4.10 & $f_{12}$ & 7.786 & 1.70 \\
\hline$f_{17}+1$ & 12.836 & 2.78 & $f_{2}$ & 10.473 & 3.47 & $f_{17}+1$ & 12.819 & 1.57 \\
\hline$f_{8}-1$ & 8.107 & 4.68 & $f_{7}-1$ & 4.988 & 4.48 & $f_{8}$ & 9.018 & 1.79 \\
\hline$f_{15}$ & 5.782 & 4.22 & $f_{15}+1$ & 6.767 & 4.23 & $f_{9}$ & 7.033 & 0.55 \\
\hline$f_{12}$ & 7.883 & 3.49 & $f_{12}$ & 7.634 & 2.99 & & & \\
\hline$f_{15}-1$ & 4.743 & 3.80 & $f_{14}-1$ & 9.817 & 3.08 & & & \\
\hline$f_{2}+1$ & 11.420 & 2.67 & & 5.201 & 2.68 & & & \\
\hline
\end{tabular}

suffered from severe alias problem. Figure 6 shows the steps of the frequency search process of the MOST data. The labels provide the exact frequency value of the dominant peak in the panel.

Not only was the dominant frequency at $13.36 \mathrm{~d}^{-1}$ confirmed but the second and third of the highest amplitudes at 10.476 and $6.477 \mathrm{~d}^{-1}$ were also definite. The fourth frequency at $12.228 \mathrm{~d}^{-1}$ can be detected without any doubt. The dominant mode and this latter frequency caused confusion in the Jørgensen et al. (1971) and
Jørgensen \& Nørgaard-Nielsen (1975) papers and in the paper of Balona (2000). The consecutive steps revealed frequencies at 6.916 and $6.070 \mathrm{~d}^{-1}$, which are similar to the frequencies found by Poretti (1989). The possible explanation that the 5.4 and $6.4 \mathrm{~d}^{-1}$ frequencies are $1 \mathrm{~d}^{-1}$ aliases was excluded here. Beside the frequency at $f_{3}=6.477 \mathrm{~d}^{-1}$, the other frequency at $f_{11}=5.498 \mathrm{~d}^{-1}$ (2nd right panel) also appeared with lower amplitude. This is another reason for a severe $\pm 1 \mathrm{~d}^{-1}$ alias. However, the frequency at $5.2 \mathrm{~d}^{-1}$ was not found here in the MOST data. The other frequency that 

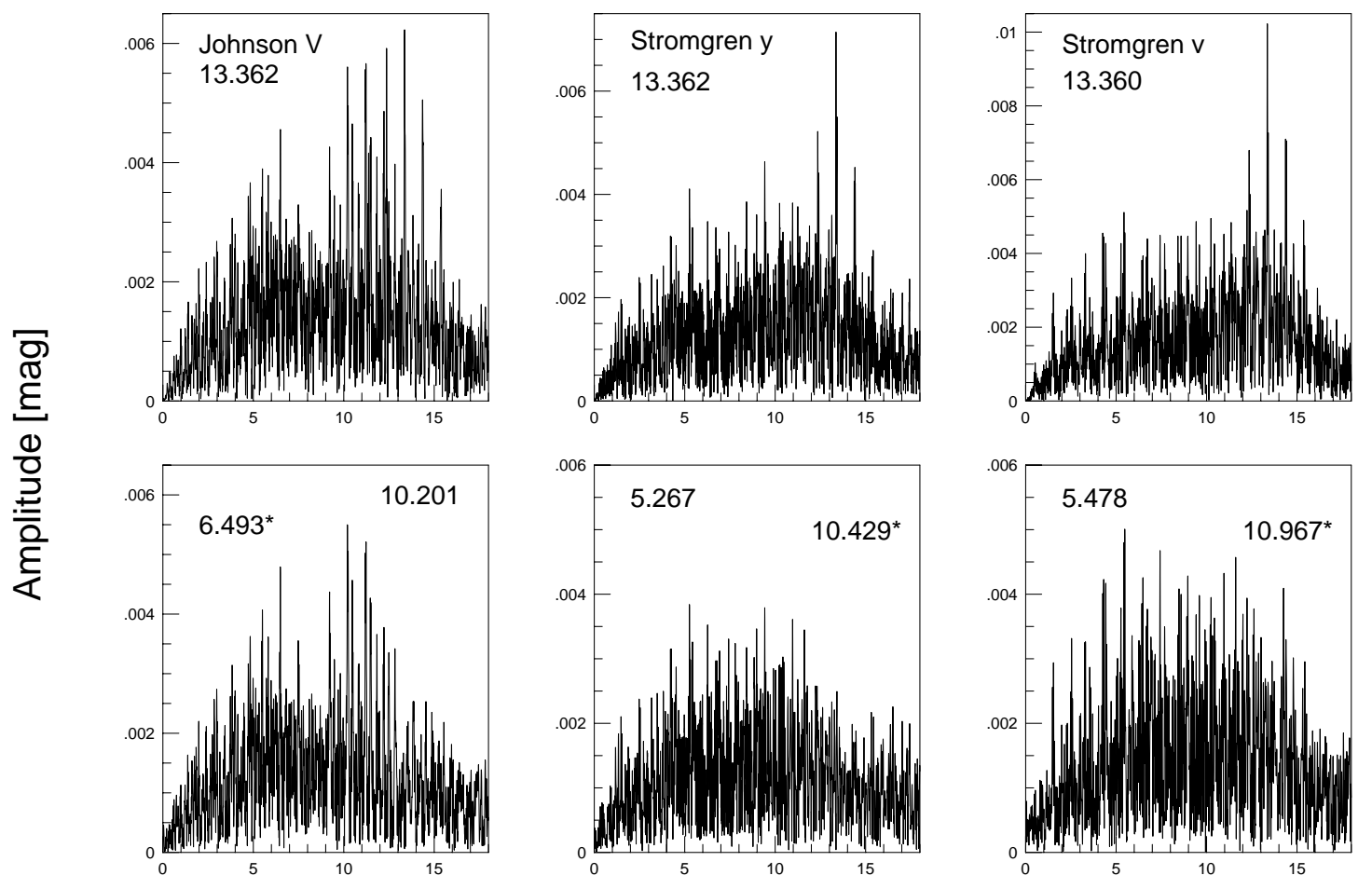

Frequency $\left[\mathrm{d}^{-1}\right]$

Figure 5. Fourier spectra in Johnson $V$, Strömgren $y$ and $v$ colours. The panels in the upper row show that the same frequency is dominant in each passband. In the bottom row the first steps of the pre-whitening process are given. One of the labels gives the peak of the highest amplitude in the spectrum. The numbers with * mean the third frequencies of the highest amplitudes in the pre-whitening process.

Table 6. Frequencies in Strömgren colours. Links to the MOST solution are given. No link means that those frequencies were not found in the MOST data. The most remarkable case is $5.267 \mathrm{~d}^{-1}$ in Strömgren $y$ colour. The Rayleigh resolution at the ground-based data is $0.028 \mathrm{~d}^{-1}$.

\begin{tabular}{|c|c|c|c|c|c|c|c|c|c|c|c|}
\hline \multicolumn{3}{|c|}{ Strömgren $u$} & \multicolumn{3}{|c|}{ Strömgren $b$} & \multicolumn{3}{|c|}{ Strömgren $v$} & \multicolumn{3}{|c|}{ Strömgren $y$} \\
\hline ID & $\begin{array}{r}\text { Freq } \\
\left(\mathrm{d}^{-1}\right)\end{array}$ & $\begin{array}{r}\text { Amp } \\
(\mathrm{mmag})\end{array}$ & ID & $\begin{array}{c}\text { Freq } \\
\left(\mathrm{d}^{-1}\right)\end{array}$ & $\begin{array}{r}\text { Amp } \\
(\mathrm{mmag})\end{array}$ & ID & $\begin{array}{r}\text { Freq } \\
\left(\mathrm{d}^{-1}\right)\end{array}$ & $\begin{array}{r}\text { Amp } \\
(\mathrm{mmag})\end{array}$ & ID & $\begin{array}{r}\text { Freq } \\
\left(\mathrm{d}^{-1}\right)\end{array}$ & $\begin{array}{r}\text { Amp } \\
\text { (mmag) }\end{array}$ \\
\hline$f_{1}$ & 13.363 & 10.48 & $f_{1}$ & 13.360 & 8.56 & $f_{1}$ & 13.360 & 11.45 & $f_{1}$ & 13.362 & 6.26 \\
\hline$f_{3}-1$ & 5.433 & 3.90 & $f_{2}$ & 10.477 & 3.22 & $f_{3}-1$ & 5.478 & 5.85 & & 5.267 & 3. \\
\hline$f_{5}+1$ & 12.624 & 4.54 & $f_{18}$ & 10.204 & 3.16 & $f_{14}$ & 10.967 & 4.62 & $f_{2}$ & 10.429 & 5.47 \\
\hline$f_{6}$ & 6.920 & 4.34 & $f_{3}-1$ & 5.429 & 3.13 & $f_{12}-1$ & 6.728 & 3.60 & $f_{3}+1$ & 7.424 & 3.54 \\
\hline & 5.267 & 3.37 & $f_{5}$ & 11.609 & 3.55 & & 6.254 & 3.50 & $f_{8}$ & 8.974 & 2.46 \\
\hline$f_{2}$ & 10.475 & 3.51 & $f_{6}$ & 6.924 & 3.72 & $f_{5}+1$ & 12.573 & 3.44 & $f_{5}$ & 11.376 & 3.91 \\
\hline$f_{14}$ & 10.962 & 2.44 & & 14.258 & 3.10 & & 14.248 & 3.37 & $f_{4}$ & 12.236 & 2.98 \\
\hline$f_{16}+1$ & 14.924 & 2.80 & & 13.233 & 2.73 & $f_{2}$ & 10.475 & 3.96 & & 10.206 & 2.81 \\
\hline$f_{3}+1$ & 7.412 & 2.84 & & 6.264 & 2.88 & $f_{7}$ & 6.089 & 3.53 & & & \\
\hline & 13.278 & 2.23 & & & & $f_{13}$ & 9.601 & 3.10 & & & \\
\hline$f_{8}+1$ & 10.191 & 1.98 & & & & & & & & & \\
\hline
\end{tabular}

shows relatively much lower amplitude is $f_{18}=10.201 \mathrm{~d}^{-1}$ that was the frequency of the second highest amplitude in Johnson $B$ and $V$ colours.

As we see in the MOST data set the $\pm 1 \mathrm{~d}^{-1}$ aliases are resolved. However, in the MOST spectrum an alias structure appears at the orbital frequency at $14.2 \mathrm{~d}^{-1}$. An interesting effect is that the sum of the frequencies at 6.477 and $7.731 \mathrm{~d}^{-1}$ is equal to $14.2 \mathrm{~d}^{-1}$, the orbital fre- quency. Pre-whitening with the higher amplitude frequency at $6.477 \mathrm{~d}^{-1}$, the rather high amplitude peak at $7.7 \mathrm{~d}^{-1}$ disappears, but after many steps we were able to localise a peak at $f_{12}=7.731 \mathrm{~d}^{-1}$ (3rd right panel). However, prewhitening with both frequencies the amplitude of the latter one increased showing that they could not be properly determined (marked by colon in Table 7). The question arises, whether it is only a remnant of the frequency subtracting 


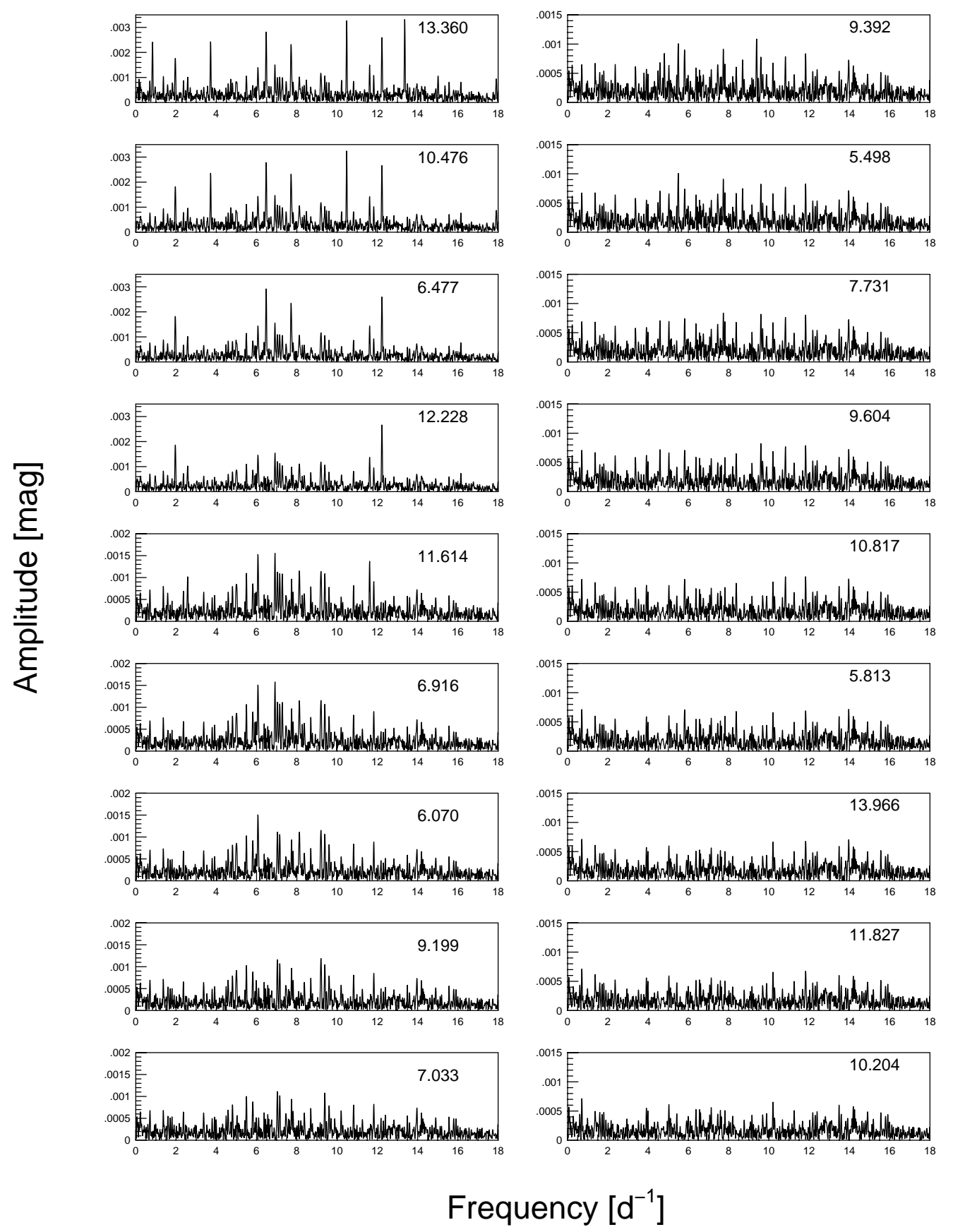

Figure 6. Steps of frequecy search in the Fourier analyses of MOST data. The amplitude scale was changed from the fifth and the tenth panel for getting a better view of the low amplitude peaks. The label marks the peak of the highest amplitude.

or it is a real frequency excited in the star. The latter version is supported by the fact that a frequency at $7.74 \mathrm{~d}^{-1}$ appeared in the analyses of Jørgensen \& Nørgaard-Nielsen (1975) and in our ground-based data sets, that are not connected to the MOST orbital frequency. Of course, the most definite answer could be obtained from a continuous data, however, unfortunately 38 Eri was not continuously observed by MOST. In some cases an algorithm for filling the gaps and getting continuous data could be an acceptable solution. Pascual-Granado, Garrido \& Suárez (2015) developed MiARMA, a new gap-filling algorithm, which is suggested to use for space data with gaps smaller than the data segments (like gaps in CoRoT light curves due to the South Atlantic Anomaly). However, predictions lose coherence rapidly when gaps are much larger than the data segments. We could not use MiARma for the shared MOST data, since the gaps are longer than the data segments.

We also found a numerical relation between the dominant mode $\left(13.360 \mathrm{~d}^{-1}\right)$ and the sum of 6.477 and $6.916 \mathrm{~d}^{-1}$ (equal to $13.393 \mathrm{~d}^{-1}$ ). Regarding the $0.034 \mathrm{~d}^{-1}$ resolution of the MOST data, the agreement is remarkable. This nearly same value to the dominant mode with the lower resolu- 

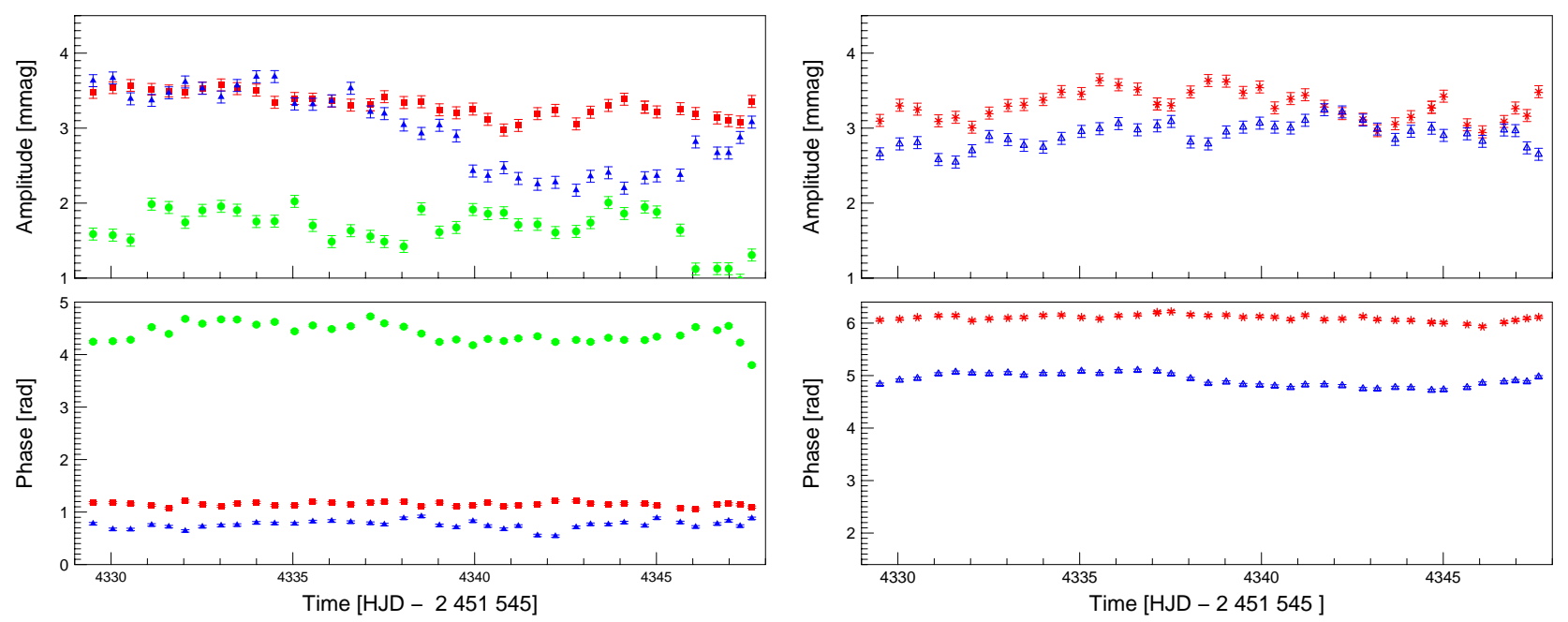

Figure 7. The amplitude variation of five high amplitude frequencies: (left) $f_{1}$ (red squares), $f_{3}$ (blue triangles), $f_{6}$ (green dots), (right) $f_{2}$ (red asterisks), $f_{4}$ (blue open triangles). The corresponding panels are at the same scales.

Table 7. Frequency solution of MOST data. Frequencies are given both in $\mathrm{d}^{-1}$ and $\mu \mathrm{Hz}$. In the phase calculation HJD 2451546.0 epoch was used. The errors of the amplitudes and phases are calculated by PERIOD04 (Lenz \& Breger 2005).

\begin{tabular}{rrrlc}
\hline ID & $\begin{array}{r}\text { Frequency } \\
\left(\mathrm{d}^{-1}\right)\end{array}$ & $\begin{array}{r}\text { Frequency } \\
(\mu \mathrm{Hz})\end{array}$ & $\begin{array}{l}\text { Amp } \\
(\mathrm{mmag})\end{array}$ & $\begin{array}{c}\text { Phase } \\
(\mathrm{rad})\end{array}$ \\
\hline$f_{1}$ & 13.360 & 154.629 & $3.38 \pm 0.07$ & $3.387 \pm 0.018$ \\
$f_{2}$ & 10.476 & 121.250 & $3.37 \pm 0.07$ & $4.008 \pm 0.021$ \\
$f_{3}$ & 6.477 & 74.965 & $2.98 \pm 0.08$ & $4.917 \pm 0.027$ \\
$f_{4}$ & 12.228 & 141.527 & $2.73 \pm 0.06$ & $5.921 \pm 0.020$ \\
$f_{5}$ & 11.614 & 134.421 & $1.37 \pm 0.07$ & $0.615 \pm 0.058$ \\
$f_{6}$ & 6.916 & 80.046 & $1.57 \pm 0.08$ & $6.068 \pm 0.052$ \\
$f_{7}$ & 6.070 & 70.255 & $1.56 \pm 0.05$ & $1.686 \pm 0.038$ \\
$f_{8}$ & 9.199 & 106.470 & $1.22 \pm 0.05$ & $5.809 \pm 0.048$ \\
$f_{9}$ & 7.033 & 81.400 & $1.13 \pm 0.08$ & $6.120 \pm 0.073$ \\
$f_{10}$ & 9.392 & 108.704 & $1.03 \pm 0.07$ & $1.485 \pm 0.057$ \\
$f_{11}$ & 5.498 & 63.634 & $0.95 \pm 0.10$ & $4.115 \pm 0.087$ \\
$f_{12}$ & 7.731 & 89.479 & $1.30: \pm 0.07$ & $4.354 \pm 0.032$ \\
$f_{13}$ & 9.604 & 111.157 & $0.88 \pm 0.12$ & $3.580 \pm 0.075$ \\
$f_{14}$ & 10.817 & 125.197 & $0.68 \pm 0.08$ & $0.152 \pm 0.090$ \\
$f_{15}$ & 5.813 & 67.280 & $0.73 \pm 0.07$ & $2.583 \pm 0.127$ \\
$f_{16}$ & 13.966 & 161.644 & $0.70 \pm 0.05$ & $2.097 \pm 0.079$ \\
$f_{17}$ & 11.827 & 136.887 & $0.71 \pm 0.09$ & $5.131 \pm 0.115$ \\
$f_{18}$ & 10.204 & 118.102 & $0.70 \pm 0.05$ & $4.620 \pm 0.083$ \\
\hline
\end{tabular}

tion of the ground-base data increased the confusion around the dominant mode of 38 Eri. The MOST data set with its better resolution can resolve these three frequencies without any doubt.

The finally accepted list of frequencies in 38 Eri are given in Table 7 . The table contains the ID of the frequencies, the frequencies both in $\mathrm{d}^{-1}$ and $\mu \mathrm{Hz}$, the amplitude and the phases to the MOST epoch (HJD-2 451545.0). These IDs are linked to the ground-based frequencies in Table 5 and Table 6 .

\subsubsection{Comparison of the ground-based and MOST frequencies}

The links in Table 5 and Table 6 remind us of the serious $\pm 1 \mathrm{~d}^{-1}$ alias problem of the ground-based observation, even though it was a multi-site international campaign. In many cases the frequencies obtained on the colour data could be connected to the +1 or $-1 \mathrm{~d}^{-1}$ alias of the MOST frequencies. However, there are frequencies in Table 6 (Strömgren colours) where no link to the MOST frequencies is given. The most interesting examples are $5.26 \mathrm{~d}^{-1}$ in $u$ and $y$ and probably its $+1 \mathrm{~d}^{-1}$ alias at $6.26 \mathrm{~d}^{-1}$ in $b$ and $v$ colours. Both frequencies in each colour, except in $y$, appear together with $5.4 \mathrm{~d}^{-1}$, which could be $f_{11}$ or the possible $-1 \mathrm{~d}^{-1}$ alias of $f_{3}$ in the list of MOST frequencies. In the Strömgren $y$ colour the $+1 \mathrm{~d}^{-1}$ alias of $f_{3}$ frequency appears. The differences in decimals, disregarding the \pm 1 alias, are $0.166,0.165,0.244$ and $0.269 \mathrm{~d}^{-1}$, which are larger than the resolution in the colour data sets. Frequencies at 5.2 and $5.4 \mathrm{~d}^{-1}$ seems to represent a pair in the ground-based data. A similarly close frequency pair appears in Johnson $B, V$ and Strömgren $u$ at $10.214 / 10.481,10.201 / 10.473$ and $10.191 / 10.475 \mathrm{~d}^{-1}$, respectively. The differences are $0.267,0.272$ and $0.284 \mathrm{~d}^{-1}$. Although these frequencies also appear in the MOST frequencies as $f_{2}$ and $f_{18}$, the later one has much lower amplitude than the frequencies around $10.2 \mathrm{~d}^{-1}$ in the groundbased data. We may speculate whether these pairs represent rotational doublets with changing amplitude at different epochs.

Slightly smaller frequency differences appear in decimals between the dominant frequency and a peak of lower amplitude, namely 13.36/13.278, 13.360/13.233 and $13.360 / 14.248 \mathrm{~d}^{-1}$ in Strömgren $u, b$ and $v$ colours. These are $0.087,0.127,0.113$ and $0.083 \mathrm{~d}^{-1}$, which are closer to the resolution. These frequencies are critical in the MOST data due to the $14.2 \mathrm{~d}^{-1}$ orbital frequency. The frequency solution in the different colour bands would suggest two possible explanations: at different epochs different frequencies are excited with different amplitudes or with the different 
passbands we are able to catch the pulsation in structurally changing layers.

The comparison of the results of the individual groundbased observing runs and the MOST run reveal that the pulsation is rather complex and we could see only a certain aspect of the complex pulsation at a certain moment. With 18 frequencies we still could not get a perfect fit (residual is $0.0025 \mathrm{mag}$ ). The light curve contains abrupt changes from extremely large amplitude cycles to cycles with very tiny amplitude (see Fig. 1). Normal beating of far-away frequencies do not show such behaviour, only the interaction of frequencies that are rather close to each other.

\subsection{Amplitude variability of $M O S T$ frequencies}

The available theoretical models, in a lack of non-linear treatment of non-radial pulsation, do not provide any prediction for the amplitude and phase variability of the excited modes. Nevertheless, the investigations of amplitude and phase variability nowadays are very common, thanks to the extended ground-based follow up (Breger 2009, 2010) and the space data of long time base (MOST, Walker et al. 2003; Matthews et al. 2004, CoRoT, Baglin 2006 and $\mathrm{Ke}$ pler, Borucki et al. 2010; Koch et al. 2010). A possible amplitude and phase variability could help to explain the frequency spectrum that is rather complicated in some cases. Amplitude variability has been published in the wide region of the HRD in the last years; for B stars interpreted as rotational modulation (Balona et al. 2015), for $\delta$ Scuti stars due to resonant mode coupling (Barceló Forteza et al. 2015), for roAp star on a shorter time-scale that was expected (Medupe et al. 2015) and even for a white dwarf as an observational consequence of a significantly crystallized stellar interior (Hermes et al. 2015). For more examples we refer for the review of Guzik et al. (2016). In a recent paper using the Kepler targets Bowman et al. (2016) presented an excellent summary on the possible cause of the amplitude and phase variation in $\delta$ Scuti stars.

Although our data do not stretch over as long a timebase as long as the Kepler data, however, the MOST observations of one month time-base gave a chance to investigate the possible amplitude and phase variability of at least the frequencies of highest amplitude in our list.

The original decision to present only the first four frequencies was evident, since they represent the same amplitude level in the range of 3.38-2.73 mmag in the MOST data. However, the numerical relation among the frequencies $\left(f_{1}, f_{3}\right.$ and $f_{6}$, discussed later) resulted the necessity of presenting the amplitude and phase of five frequencies. The 29.5 day long time-base was divided into eight-day long segments shifted by 0.5 days to follow the variability as much as possible, although the points are not independent. PERIOD04 (Lenz \& Breger 2005) was used to get the amplitude and phase values and the uncertainties for the segments. Figure 7 shows the amplitude (top panel) and phase (bottom panel) variability of five frequencies. The error bars represent similar uncertainties that are given in Table 7 . The time base shown in Figure 7 is shorter than the duration of the MOST observation. We used only the solution for the complete eight-day long segments. In addition, due to the larger gaps in the data much larger error bars resulted at the end, which we omitted from the panel. We present three frequencies $\left(f_{1}=13.360, f_{3}=6.477\right.$, and $\left.f_{6}=6.916 \mathrm{~d}^{-1}\right)$ in the left side panels and two frequencies $\left(f_{2}=10.476\right.$ and $f_{4}=12.228 \mathrm{~d}^{-1}$ ) in the right panels.

The constant phases show that the not too long time base, therefore not too high resolution, allows the determination of the possible variability. We regarded the amplitudes of $f_{2}$ and $f_{4}$ in the right side panel as constant amplitudes based on two criteria. First, a numerical test on the data generated with four sinus waves on the time distribution of the MOST data, resulted in a 5 percent scatter around the constant amplitude and phase, due to the gaped data distribution. The amplitude variation of $f_{2}$ and $f_{4}$ were not larger than 5 percent around the mean level. Secondly, following the criteria of constancy given by Bowman et al. (2016), namely, a frequency exhibits significant amplitude variability if at least half of its amplitude bins deviate more than $\pm 5 \sigma$ from its mean value we concluded that $f_{2}=10.476$ and $f_{4}=12.228 \mathrm{~d}^{-1}$ do not show amplitude variability.

The three frequencies on the left side are separately presented, because $f_{1} \approx f_{3}+f_{6}$, which means a necessary criteria for the linear combination or resonant mode coupling. However, the phase curves are constant and an amplitude variability higher than the $5 \sigma$ criteria is valid only for $f_{3}=6.477 \mathrm{~d}^{-1}$. We have three possibly connected frequencies, but only a single amplitude variation.

According to Bowman et al. (2016) some amplitude variation can be explained by beating of two close frequencies (Breger et al. 2009) or the mode coupling mechanism (Breger 2000b). Breger \& Bischof (2002) showed that pairs of close-frequency modes were found near the expected frequencies of radial modes. In the mode identification section, the $f_{3}=6.477 \mathrm{~d}^{-1}$ frequency is a possible candidate for being a radial mode according to the period ratio of two MOST frequencies, discussed later.

Both Breger \& Bischof (2002) and Bowman et al. (2016) used the $0.01 \mathrm{~d}^{-1}$ value as a criterion for the frequency separation of the two closely spaced frequencies. Due to the resolution of our MOST data $\left(0.034 \mathrm{~d}^{-1}\right)$, we could not resolve two frequencies as closely spaced as the criterion. We could not exclude the cause of the beating for the amplitude variation of $f_{3}=6.477 \mathrm{~d}^{-1}$. If we take the amplitude variation as a periodic one with $\mathrm{a} \approx 20$ day period, the necessary frequency difference between $f_{3}$ and the unresolved frequency is $\approx 0.05 \mathrm{~d}^{-1}$.

In principle, we should be able to resolve such a close frequency to $f_{3}=6.477 \mathrm{~d}^{-1}$. Instead of a close pair we found a frequency at 6.2 (or 5.2) $\mathrm{d}^{-1}$ appearing in the groundbased data, but not in the MOST data. Such a far-away frequency does not result in amplitude variation. In addition, the beating of a pair of pulsation mode frequencies, that are close and resolved, appears as periodic amplitude modulation with a characteristic sharp change in phase at the epoch of minimum amplitude for each frequency in the pair (Breger \& Pamyatnykh 2006). However, we have a constant phase, so we conclude that the amplitude change of $f_{3}=6.477 \mathrm{~d}^{-1}$ is not caused by beating.

The non-linearity and mode coupling are worthwhile to check, because three frequencies satisfy the resonance criteria for both the combination frequency and the mode coupling.

$f_{1} \approx f_{3}+f_{6}$ 
where $f_{1}$ is the child mode and $f_{3}$ and $f_{6}$ are the parent modes. The difference between $f_{1}$ and the sum is $0.03 \mathrm{~d}^{-1}$ that is near to our resolution, but the criterion is satisfied. According to the model of Breger \& Montgomery (2014), the amplitude of the child mode is a product of the two modes as

$A_{1}=\mu_{\mathrm{c}} A_{3} A_{6}$

in our case, and the phase relation is

$\phi_{1}=\phi_{3} \pm \phi_{6}$.

Using $A_{1}=3.38, A_{3}=2.98$, and $A_{6}=1.57$ mmags from Table 7 , we obtained $\mu_{\mathrm{c}}=0.722$.

The value of $\mu_{\mathrm{c}}$ characterizes the type of connection between the frequencies. If, $\mu_{\mathrm{c}} \leq 0.01$ this implies weak coupling and favours a non-linear distortion model producing a combination frequency. A value $\mu_{\mathrm{c}}>0.1$ implies a strong coupling and favours resonant mode coupling. However, in the mode coupling hypothesis, it is required that all three members of a family are variable in amplitude, so that the child mode can be identified. In our case, only one parent mode exhibits amplitude change. In addition, $\phi_{1}=3.387$, $\phi_{3}=4.917$, and $\phi_{6}=6.068$ radians do not fulfil the phase relation of the resonant coupling. In this family hypothesis, only one frequency has variability and only in amplitude. These arguments exclude that resonant mode coupling is going on in 38 Eri. What remains for possible explanation?

Although the sum of $f_{3}=6.477$ and $f_{12}=7.731 \mathrm{~d}^{-1}$ equals to the MOST orbital frequency, $f_{\text {orb }}=14.2 \mathrm{~d}^{-1}$, this connection can only slightly modify the amplitudes if they are determined at the same time, but it can not cause an amplitude variability. Bowman et al. (2016) conjectured for the pure amplitude modulation that it could be caused by variable driving and/or damping within the star.

\subsection{Regularity between the frequencies}

We know two facts for rotating $\delta$ Scuti stars in general. They pulsate in many non-radial modes. Due to the rotation the non-radial modes split in the simplest cases into equidistant triplets or multiplets. The first-order effect (Ledoux 1951), the second-order effect (Vorontsov 1981, 1983; Dziembowski \& Goode 1992), and the third-order effect (Soufi, Goupil \& Dziembowski 1998) were investigated theoretically in the frame of the perturbative theory. Later, investigation of the intermediate and fast rotating stars, using a different approach, were carried out (Lignières et al. 2006; Roxburgh 2006; Lignières \& Georgeot 2008, 2009; Lignières et al. 2010; Reese, Lignières \& Rieutord 2008; Reese et al. 2009). In recent years the frequency pattern of the fast rotating stars combined with theoretical investigation were used to find stellar density (Suárez et al. 2014; García Hernández et al. 2015), and accurate surface gravity (García Hernández et al. 2017). The frequency regularities help in the multi-colour mode identification (Reese et al. 2017) and can be characterized by few parameters, for example $\delta \nu$ (Moya et al. 2017), which might play a role in the seismic indices for $\delta$ Scuti stars (Michel et al. 2017).

The search for regular spacing in $\delta$ Scuti stars has a long history, too, starting from the histogram method (Breger et al. 1999) to the Fourier transform method used at first for ground based data (Handler et al. 1997), later for the space data producing echelle diagrams (García Hernández et al. 2009, 2013, 2015).

Two dedicated approaches appeared in the last two years. Both of them looked not only for a spacing value, but also sequences of regular spacing in different region of spacing. One of them originally concentrates on the distribution of the overtones with a certain $l$ value and the shift of the sequences with different $l$ values (Paparó et al. 2013, $2016 \mathrm{a}, \mathrm{b})$. The existence of the sequences with similar spacing (in the 1.5-3.6 $\mathrm{d}^{-1}$ region) allowing a tolerance level has been confirmed (Paparó et al. 2016b). However, in some cases the pure sequence of the modes with the same $l$ value are influenced by the rotation split frequencies (see the test case of FG Vir in Paparó et al. 2016a).

The other approach searches for regularities caused by rotational splitting (Chen et al. 2016, 2017; Chen \& Li 2017). The final goal of all investigation on the regular spacing(s) is the same: to solve the problem of mode identification in $\delta$ Scuti stars, to reach the level of asteroseismology for stars pulsating in the non-asymptotic regime. We present cases here at the first time where the new observables derived from the two methods are compared, looking for possible higher level regularities.

\subsubsection{Rotation splitting}

According to the modelling (Balona 2000), 38 Eri is in a shell hydrogen burning stage of evolution. This star fits the group of evolved stars (EE Cam, HD 50844 and CoRoT 102749568), where the approximate formulae for the highorder g-modes (derived by Brickhill 1975 and Winget et al. 1991 for white dwarfs) are applied for the $\delta$ Scuti stars' mixed modes, that is, for modes showing p-mode character in the envelope and g-mode character in the inner region. White dwarfs are pulsating in g-modes in the envelope, while the g-modes in $\delta$ Scuti stars have low amplitude in the envelope. Although Dziembowski \& Goode (1992) mentioned that g-mode asymptotics are relevant also for $\delta$ Scuti stars, we checked the validity of eq. 4 for 38 Eri. We computed one non-rotating evolutionary track using the MESA code (Paxton et al. 2011, 2013, 2015). We used solar metallicity and mixing length parameter $\alpha_{\mathrm{MLT}}=0.5$ following the presciption of García Hernández et al. (2009) for $\delta$ Scuti stars. The track was computed with $M=2 \mathrm{M}_{\odot}$ (Balona 2000) and was evolved up to reaching the observed surface gravity. All the models fitting the observed parameters (Fig. 8) showed a cut-off frequency above $3000 \mu \mathrm{Hz}$ $\left(=259.2 \mathrm{~d}^{-1}\right)$ and a Brunt-Väisälä frequency above $160 \mathrm{~d}^{-1}$ in the convective core (Fig. 9). The latter value demonstrates that 38 Eri fulfills the conditions to apply eq. 4 , i. e., that the rotational frequency is lower than the observed frequencies and that these are lower than the Lamb and Brunt-Väisälä frequencies.

We applied the rotational splitting method for 38 Eri. The approximate formula of the rotational splitting $\delta \nu_{l, n}$ and the rotational period $\left(P_{\text {rot }}\right)$ for the high order g-modes is

$m \delta \nu_{l, m}=\frac{m}{P_{\text {rot }}}\left[1-\frac{1}{l(l+1)}\right]$

where $l, n$, and $m$ are the quantum numbers of the pulsation modes. We did not apply the rotational splitting method in 


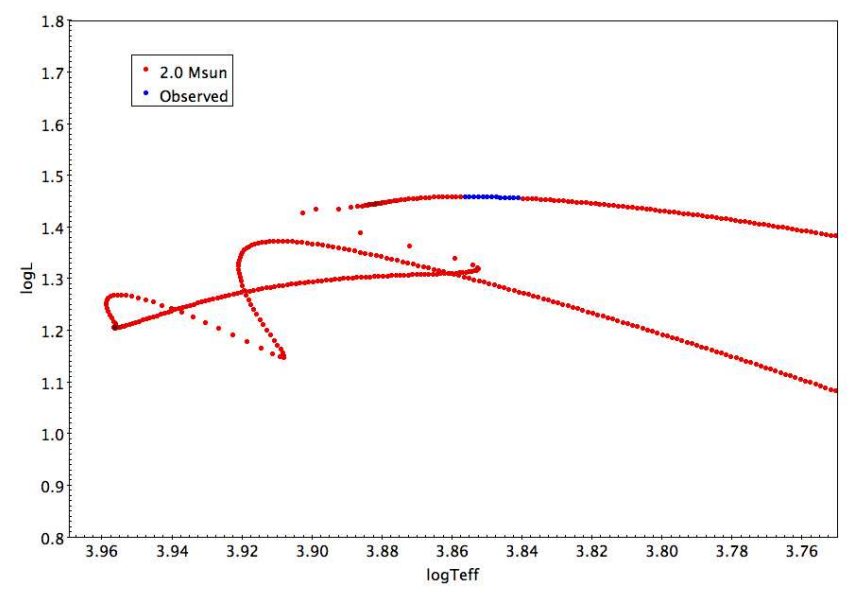

Figure 8. Evolutionary status of 38 Eri. HR diagram of an evolutionary track with $M=2 \mathrm{M}_{\odot}$. Red dots show the complete track from the pre-main sequence to the hydrogen-shell burning phase. Blue dots correspond to the models in the range of observed $T_{\text {eff }}$ and $\log g$.

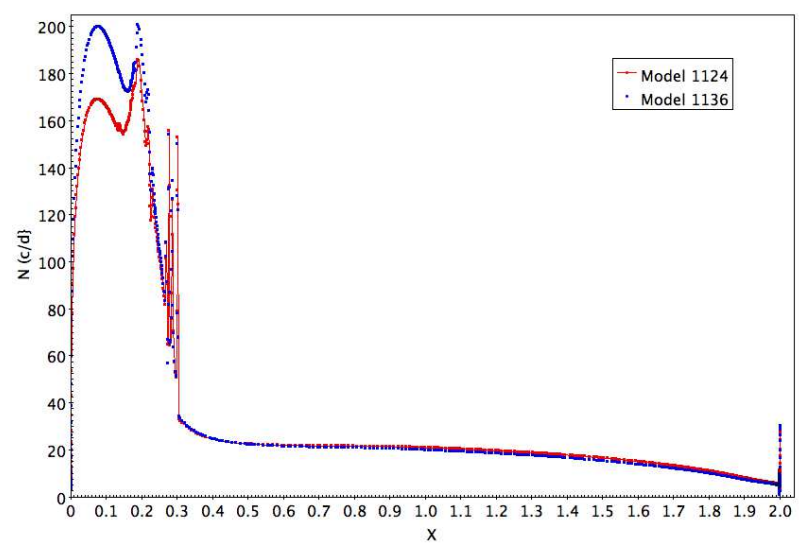

Figure 9. Lamb and Brunt-Väisälä frequencies of 38 Eri. BruntVäisälä frequency $(N)$ versus mass for the limiting models in the range of the observed $T_{\text {eff }}$ and $\log g$. Red dots correspond to the less evolved model and blue dots correspond to the most evolved one. Both models show $N$ values in the core well above the observed frequencies.

the original way. We did not search for the 1-20 $\mu \mathrm{Hz}(0.0864-$ $\left.1.728 \mathrm{~d}^{-1}\right)$ region as Chen et al. (2016, 2017); Chen \& Li (2017) did, but we did only a visual search among the frequencies (only 18), but all of the small spacings were calculated. We noticed that nearby frequencies have a spacing around $0.2 \mathrm{~d}^{-1}$ or its multiple value. These values were not exactly the same in each case (we may not expect it), so we could not pick up a certain value for the rotatinal splitting. However, we recognezed that the most frequent smallest unit of the spacing is near the expected rotational splitting, if we suppose that the inclination angle is close to $90^{\circ}$ and we use $P_{\text {rot }}$ obtained from the spectroscopic $v \sin i$. According to our assumption, if the inclination is really close to $90^{\circ}$, then we find spacing with these and it proves that our assumption is not completely wrong. The rotational period according to Balona (2000) and McDonald et al. (2017) are $P_{\text {rot }}<1.9$ and $<1.8$ day for 38 Eri (see in Table 1$)$. The
Table 8. Possible rotational splittings. The spacing values were searched according to Eq. 4.

\begin{tabular}{llllll}
\hline Multiplet & ID & $\begin{array}{l}\text { Freq } \\
\left(\mathrm{d}^{-1}\right)\end{array}$ & $\begin{array}{l}\delta \nu \\
\left(\mathrm{d}^{-1}\right)\end{array}$ & $l$ & $m$ \\
\hline 1 & $f_{15}$ & 5.813 & 0.257 & 1 & $-1 / 0$ \\
& $f_{7}$ & 6.070 & & 1 & $0 /+1$ \\
& & & & 1 & $-1 / 0$ \\
2 & $f_{2}$ & 10.476 & 0.272 & 1 & $0 /+1$ \\
& $f_{18}$ & 10.204 & & & \\
& & & & 2 & $-1 / 0$ \\
3 & $f_{3}$ & 6.476 & 0.439 & 2 & $0 /+1$ \\
& $f_{6}$ & 6.916 & $2 * 0.442$ & 2 & +2 \\
& $f_{12}$ & 7.731 & & & \\
4 & & & & 2 & -2 \\
& $f_{2}$ & 10.476 & $2 * 0.436$ & 2 & 0 \\
& $f_{13}$ & 9.604 & & & \\
5 & & & & 3 & $-2 / 0$ \\
& $f_{11}$ & 5.498 & $2 * 0.490$ & 3 & $0 /+2$ \\
6 & $f_{3}$ & 6.477 & & & \\
& & & & 3 & $-2 / 0$ \\
& $f_{9}$ & 7.033 & $2 * 0.482$ & 3 & $0 /+2$ \\
\hline
\end{tabular}

Table 9. Sequence search result. Four sequences were identified with near similar spacing. The averaged spacings are given in the last column. The frequencies are given in $\mathrm{d}^{-1}$.

\begin{tabular}{lcrrccc}
\hline No & Freq & Freq & Freq & Freq & Freq & Av. spacing \\
\hline I & 5.498 & 6.916 & 10.476 & 12.228 & 13.966 & 1.672 \\
II & 5.813 & 7.731 & 9.604 & 11.614 & 13.360 & 1.887 \\
III & 6.477 & 10.204 & 11.827 & & & 1.744 \\
IV & 6.070 & 9.199 & 10.817 & & & 1.591 \\
\hline
\end{tabular}

expected rotational splittings according to the formula are $\delta \nu_{1}>0.263(0.278) \mathrm{d}^{-1}$ for $l=1, \delta \nu_{2}>0.438(0.463) \mathrm{d}^{-1}$ for $l=2$, and $\delta \nu_{3}>0.482(0.509)$ for $l=3$. The expected ratios are $\delta \nu_{1} / \delta \nu_{2}=0.6, \delta \nu_{3} / \delta \nu_{2}=1.1$ which confirms the relation derived by Winget et al. (1991), namely, $\delta \nu_{n, l=1}: \delta \nu_{n, l=2}: \delta \nu_{n, l=3}=0.6: 1: 1.1$. We searched for triplets and multiplets with these spacings among the frequencies given in Table 7 .

In accordance with the small number of frequencies, it is not surprising that we did not find clear triplets as in the published papers, but we found only some doublets and incomplete multiplets. The possible rotational splittings are given in Table 8.

The search for rotational splitting resulted in two doublets for $l=1$, two incomplete multiplets for $l=2$, and two doublets for $l=3$. The $m$ values in Table 8 are tentatively given; there is no strong base for the identification, since we do not have complete triplets. Averaging the observed spacing gives $\delta \nu_{1}=0.265, \delta \nu_{2}=0.439$, and $\delta \nu_{3}=0.486$. The three values are in good agreement with the theoretically expected values confirming that 38 Eri has a high inclination angle. The observed ratios are $\delta \nu_{1} / \delta \nu_{2}=0.604$ and $\delta \nu_{3} / \delta \nu_{2}=1.107$. Although the limited number of frequencies (due to the short time base) did not allow us to get triplets and to identify the exact $m$ values, but we concluded from the rotational splitting method that $l=1, l=2$, and 
Table 10. Multi-colour amplitude ratios and phase differences given for mode identification. The averaged values of the phase differences are in the last column. The deviations from the average are given in brackets. These values were used as error bars in Figure 10.

\begin{tabular}{rrrrrrrrr}
\hline No & $\begin{array}{r}\text { Freq } \\
\left(\mathrm{d}^{-1}\right)\end{array}$ & $\begin{array}{r}\phi_{b}-\phi_{y} \\
(\mathrm{deg})\end{array}$ & $A_{b} / A_{y}$ & $\begin{array}{r}\phi_{v}-\phi_{y} \\
(\mathrm{deg})\end{array}$ & $A_{v} / A_{y}$ & $\begin{array}{r}\phi_{B}-\phi_{V} \\
(\mathrm{deg})\end{array}$ & $A_{B} / A_{V}$ & Average \\
\hline$f_{1}$ & 13.360 & $-4.13(-0.26)$ & 1.34 & $-2.87(+1.0)$ & 1.523 & $-4.61(-0.74)$ & 1.514 & -3.87 \\
$f_{2}$ & 10.476 & $-4.22(-3.17)$ & 1.132 & $+4.76(+5.81)$ & 1.044 & $-1.59(-0.54)$ & 1.316 & -1.05 \\
$f_{3}$ & 6.47 & $-5.85(-0.24)$ & 1.038 & $-0.80(+4.81)$ & 1.629 & $-10.19(-4.58)$ & 1.827 & -5.61 \\
$f_{4}$ & 12.228 & $-26.11(-3.84)$ & 1.077 & $-18.44(+3.84)$ & 1.875 & & & -22.28 \\
$f_{5}$ & 11.614 & $-15.26(-1.01)$ & 1.310 & $-13.24(+1.01)$ & 1.840 & & & -14.25 \\
$f_{6}$ & 6.916 & $-6.12(-3.28)$ & 1.154 & $+0.44(+3.28)$ & 1.114 & & -2.84 \\
$f_{8}$ & 9.199 & $-20.70(-7.47)$ & 0.904 & $-5.26(+7.47)$ & 1.092 & $-13.72(-0.49)$ & 2.013 & -13.23 \\
$f_{11}$ & 5.498 & $+3.48(+0.46)$ & 1.022 & $+15.92(+12.90)$ & 1.709 & $-10.33(-13.35)$ & 1.581 & +3.02 \\
$f_{13}$ & 9.604 & $-22.59(+1.18)$ & 1.294 & $-24.94(-1.18)$ & 1.864 & & & -23.77 \\
$f_{15}$ & 5.813 & $+2.48(-3.36)$ & 0.577 & $+11.81(+5.97)$ & 0.890 & $+3.23(-2.61)$ & 0.973 & +5.84 \\
$f_{18}$ & 10.204 & $+4.93(+1.91)$ & 1.212 & $+2.75(-0.26)$ & 1.261 & $+1.39(-1.63)$ & 1.457 & +3.02 \\
\hline
\end{tabular}

Table 11. The rotational split values for the post-main sequence stars, based partly on Chen et al. (2016); Chen \& Li (2017); Chen et al. (2017) and the present investigation.

\begin{tabular}{llll}
\hline Star & $\delta \nu_{1}$ & $\delta \nu_{2}$ & $\delta \nu_{3}$ \\
\hline 38 Eri & 0.265 & 0.439 & 0.486 \\
HD 50844 & 0.210 & 0.346 & 0.386 \\
EE Cam & 0.281 & 0.467 & \\
CoRoT 102749568 & 0.384 & 0.643 & 0.706 \\
\hline
\end{tabular}

$l=3$ modes are excited in 38 Eri according to this method. Since the frequencies of the highest amplitudes are included in the non-radial doublets/multiplets, except $f_{1}=13.360$, we may exclude that the $f_{2}=10.476, f_{3}=6.476$, and $f_{4}=12.228 \mathrm{~d}^{-1}$ frequencies are radial modes. The frequency at $f_{1}=13.360 \mathrm{~d}^{-1}$ could be a radial mode. Checking the amplitude ratios in the doublets and the triplet, we found small amplitude for the possible $m=0$ and larger amplitude for the $m= \pm 1$ frequencies. Similar amplitudes appeared in the triplet with $l=2$, as it was predicted by Gizon \& Solanki (2003) for high inclination.

However, a definite weakness of our result is that two frequencies $\left(f_{2}\right.$, and $\left.f_{3}\right)$ belong to doublets/multiplets with $l=1$ and $l=2$, or $l=2$ and $l=3$. Probably we missed some members of overlaping multiplets due to the small number of frequencies. In addition, the reviewer called our attention to the fact that two single spacings, $f_{14}$ $f_{2}=0.341$ and $f_{7}-f_{11}=0.572 \mathrm{~d}^{-1}$, have 0.6 ratio. In this case the $P_{\text {rot }}=1.466 \mathrm{~d}^{-1}$ and the inclination is around 50 degree. However, no single spacing $\left(0.625 \mathrm{~d}^{-1}\right)$ was found that could satisfy the other expected ratio. Although it is a charming possibility to find the inclination of the rotation from the spacings, but in my view the general behaviour of the rotation is not reflected by a single spacing. Nevertherless, observables resulted in the rotational splitting method for 38 Eri and the cases presented by Chen et al. (2016, 2017); Chen \& Li (2017) give a rather definite spacing range, presented in Table 11. As Dziembowski \& Goode (1992) stated, the g-mode asymptotic seems to work for the post mainsequence stars.

\subsubsection{Sequence search}

We also applied the other approach, the sequence search method (SSA, Paparó et al. 2016a,b) for the 18 frequencies of 38 Eri. A visual inspection, concentrating on larger spacings, resulted in four sequences, each of them shifted to each other. The sequences are given in Table 9. Sixteen frequencies are included in the four sequences out of the 18 MOST frequencies. There are two observables that could be derived from the frequencies. The average spacing of each sequence is given in the last column of Table 9. Averaging these values, we get $\Delta \alpha=1.724 \pm 0.092 \mathrm{~d}^{-1}$. This is one of the observables of the SSA method.

Following the conclusion on the test case of FG Vir published in Paparó et al. (2016a), the $\Delta \alpha$ could be a combination of the large separation and the rotational frequency. In this case the sequences do not contain eigenmodes with the same $l$ value, but a mixture of eigenmodes and split modes due to the rotation, although we would like to have a method for deriving the large separation for large amount of $\delta$ Scuti stars presented by the space missions (MOST, CoRoT, Kepler and the forthcoming TESS and PLATO). Of course, we can theoretically derive it if we do modelling for each star, however, it is very time consuming. In our working hypothesis, the comparison of the observables may yield to higher level regularities. The numerical connection between the two methods is the following: $\Delta \alpha=6.53 \cdot \delta \nu_{1}=3.95 \cdot \delta \nu_{2}=3.58 \cdot \delta \nu_{3}$. These relations do not show any higher level regularity, at least that we could easily explain from a single case. The co-efficients reflect the asymptotic rotational split ratio for different $l$ values. It would be worthwhile to check for larger sample, whether the co-efficients are similar for each cases or not. Similar values would suggest that the large spacing obtained by SSA and the rotational split values are not independent of each other. At this moment we only conclude that the members of the rotatinal splitting belong to different sequences. This means that the large spacing of 38 Eri is the combination of the rotational frequency and the large separation.

Nonetheless, we used this spacing and the scaling relation in García Hernández et al. (2017) to calculate the mean densities and surface gravities for 38 Eri. We assumed that the spacing is just the large separation, since the rotational splitting is relatively small. We aim to make an 
Table 12. The ratios of the shift of the sequences to the observed rotational split of 38 Eri. We calculated the ratios for both directions, forward and backward.

\begin{tabular}{lccclccc}
\hline Seq. & $\delta \nu_{1}$ & $\delta \nu_{2}$ & $\delta \nu_{3}$ & Seq. & $\delta \nu_{1}$ & $\delta \nu_{2}$ & $\delta \nu_{3}$ \\
\hline II-I & 3.21 & 1.94 & 1.75 & I-II & 3.01 & 1.82 & 1.64 \\
IV-I & 2.65 & 1.60 & 1.44 & I-IV & 4.45 & 2.68 & 2.41 \\
III-I & 5.00 & 3.02 & 2.73 & I-III & 1.40 & 0.84 & 0.76 \\
IV-II & 3.69 & 2.23 & 2.01 & II-IV & 3.60 & 2.17 & 1.96 \\
III-IV & 3.04 & 1.83 & 1.66 & IV-III & 3.72 & 2.25 & 2.03 \\
III-II & 1.86 & 1.12 & 1.01 & II-III & 5.28 & 3.19 & 2.88 \\
\hline
\end{tabular}

estimation of these quantities, testing the validity of the scaling relation. The mean density found in this way is $\bar{\rho}=[0.0394,0.0554] \mathrm{gcm}^{-3}$. These are values obtained with the extreme cases of the spacings (see Table 9). Using a large range in masses, $M=[1,3] \mathrm{M}_{\odot}$, the estimated surface gravity is $\log g=[3.40,3.66] \mathrm{cgs}$. This is in agreement with the quantities given by Balona (2000) and McDonald et al. (2017). Moreover, taken a mean value of the four spacings and assuming a mass of $M=2 \mathrm{M}_{\odot}$, then $\log g=3.53$, perfectly matches Balona's and McDonald et al.'s values.

In the sequence search method not only is the (tentatively called) large separation an important parameter, but also the shift of the sequences to each other are. In the asymptotic regime of the non-rotating pulsating stars the sequence of eigenmodes with different $l$ are systematically shifted to the radial modes $(l=0)$. Paparó et al. (2016b) proved for $90 \delta$ Scuti stars that in non-asymptotic cases the shifts do not show a simple regularity, but we do not have any theoretical prediction for the shifts. However, the shift of the members of a sequence to the members of the other sequence could be derived, as a new observable. For example, according to Table 9 the shift of the II to I sequences are calculated as the average of 5.813-5.498, 7.731-6.916, 11.614-10.476 and 13.360-12.228 frequency differences. We compared the shifts, calculated forward (II-I) and backward (I-II) to the observed rotational split of 38 Eri. The ratios for both forward and backward are given in Table 12 .

As Table 12 shows, except the shift of IV-I and I-IV, all shifts are connected by an integer times $(2,3,5)$ to the rotational split of $l=1,2$ and 3 , but the explanation needs more cases. The two methods definitely show two different aspects of the underlying physical processes. For the completeness we checked the ratio of the rotational splits for $i=50$ degree to the shifts of the sequences. We have less cases with integer ratios, but we have some. The shift of IV-I is 2.06 times $\delta \nu_{1}$, I-IV is 2.06 times $\delta \nu_{2}$, III-I is 1.09 times $\delta \nu_{1}$, and II-III is 4.10 times $\delta \nu_{1}$. The integer values are also less precise.

An additional confirmation of the connection is supplied by CoRoT 102749568. The ratios of the rotational splitting values (Chen et al. 2017), presented in Table 11. and the independent shifts (table 6 of Paparó et al. 2013) of the $l=$ 0,1 and 2 sequences were calculated.

The comparison of the rotational splitting and the shift of the members of the same sequences also resulted in an almost integer ratio. The differences between the same radial order of the $l=0$ and $l=1$ sequences were 2,3 and 4 times the value of $\delta \nu_{1}$. More than a single ratio was obtained, since in this case we compared the independent shifts, not the averaged value, to the rotatinal splitting values. The consecutive radial orders in the $l=0$ and $l=1$ sequences differ by two times $\delta \nu_{3}$. The consecutive radial orders of the $l=2$ and $l=0$ sequences differ by five times $\delta \nu_{1}$ or three times $\delta \nu_{2}$. It seems that pulsation and rotation have a strong effect on each other that it is not easy to disentangle, but maybe these methods take us closer to the solution.

\section{MODE IDENTIFICATION}

\subsection{Period ratio and pulsation constant}

Since the systematic theoretical calculation of the period ratios (Stellingwerf 1979) and the pulsation constants (Fitch 1981) for $\delta$ Scuti stars, both observables were widely used in the $80 \mathrm{~s}$ for mode identification (see Breger 1979; Poretti, Mantegazza \& Antonello 1987; Poretti, Antonello \& Mantegazza 1988; Poretti 1989). In radially pulsating stars the period ratios of the excited modes are good tools for mode identification. The ratio of the radial overtones yield certain values that we can use for mode identification. The theoretical radial period ratios for the nonradially pulsating $\delta$ Scuti stars calculated by Stellingwerf (1979) are 0.756-0.789 for $P_{1} / P_{0}, 0.611-0.632$ for $P_{2} / P_{0}$ and 0.500-0.525 for $P_{3} / P_{0}$, where $P_{0}, P_{1}, P_{2}$ and $P_{3}$ are periods of the radial fundamental, first, second and third radial overtone modes.

The subsequent calculations on the dependence of the period ratios on the metallicity and the rotation (Suárez \& Garrido 2006; Suárez, Garrido \& Goupil 2006; Suárez, Garrido \& Moya 2007) revealed that both the higher metallicity and the higher rotational velocity increase the period ratios with around $10^{-3}$. The typical $P_{1} / P_{0}$ values found for main sequence Pop I stars are in the range of $[0.772,0.776]$. Unfortunately, there is a mix-up between the two effects, so the usefulness of the period ratios for mode identification is not as straightforward as it was thought in the early 80 s. In the lack of new period ratios for higher radial overtones, we used the rather wide range of (Stellingwerf 1979) for checking the modes of 38 Eri.

The $f_{3} / f_{2}=0.618$ ratio suggests that $f_{3}=6.477 \mathrm{~d}^{-1}$ is the radial fundamental and $f_{2}=10.476 \mathrm{~d}^{-1}$ is the radial second overtone. Accepting $f_{3}=6.477 \mathrm{~d}^{-1}$ as a radial fundamental mode, the $f_{3} / f_{1}=0.485$ and $f_{3} / f_{4}=0.530$ ratios rule out that either $f_{1}$ or $f_{4}$ would be the third radial overtone. However, the $f_{2} / f_{1}=0.784$ ratio suggests that $f_{2}=10.476 \mathrm{~d}^{-1}$ is a radial fundamental and $f_{1}=13.360 \mathrm{~d}^{-1}$ is the radial first overtone according to Stellingwerf's wider range, however, the more severe $[0.772,0.776]$ range of Suárez \& Garrido (2006) rules out that $f_{1}$ and $f_{2}$ would be radial modes.

We calculated the $Q$ value for the four frequencies, $f_{1}$, $f_{2}, f_{3}$ and $f_{4}$, using the empirical relation

$\log Q=\log P+\frac{1}{2} \log g+\frac{1}{10} M_{\mathrm{bol}}+\log T_{\text {eff }}-6.454$

given by Bowman et al. (2016) and both sets of the physical parameters from Table 1 . The typical values of pulsation constants for fundamental, first and second overtone radial p-modes in $\delta$ Scuti stars lie in the range $0.022 \leq Q \leq 0.033$ (Breger \& Bregman 1975). The empirical relation resulted in $Q=0.032$ or 0.029 for $f_{3}=6.477 \mathrm{~d}^{-1}$ allowing the possibility for being a radial mode. All the other frequencies 

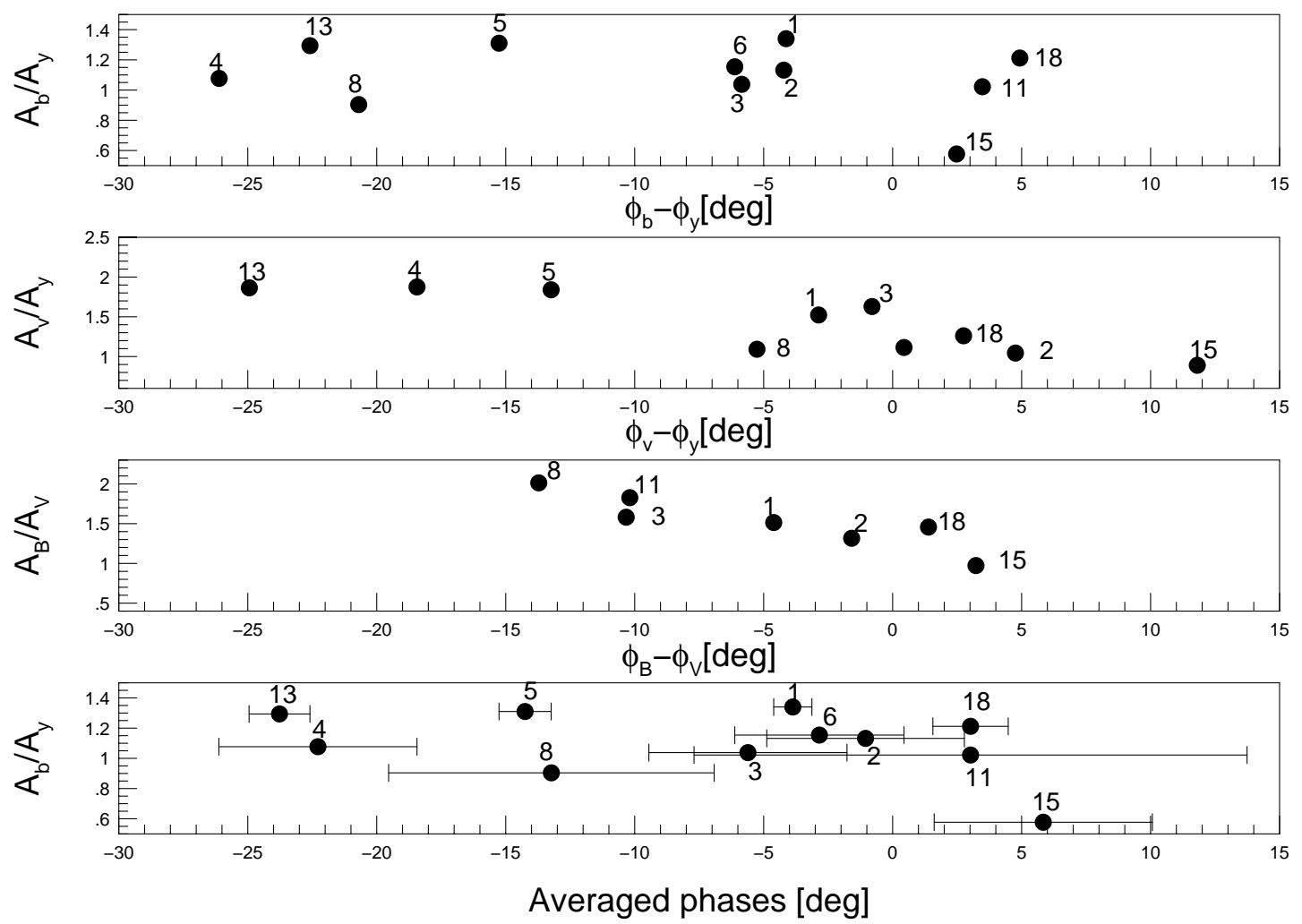

Figure 10. Discriminative panels for mode identification on Strömgren $b, v, y$, Johnson $B$ and $V$ colours. In the fourth panel the average of the phase differences of the previous panels is shown versus the amplitude ratios of the first panel. Each amplitude ratios and phase differences are given in Table 10. The deviation of the phase differences from the averaged value are given in bracket. Frequences are separated in three different groups especially in the top panel. The location of the same frequencies can be followed from panel to panel at the same place. An estimation of error in the phase differences were made using the three two-colour diagrams. The frequencies are well-separated in phase differences in the fourth panel. The indentification of frequencies are based on the general trend of the theoretical investigation, namely, that the phase differences change from right to left for the $l=0,1$ and 2 modes. The identification obtained in our hybrid methods is given in Table 13.

of highest amplitude are out of the range. A further check resulted in $Q=0.020$ or 0.019 for $f_{15}$ and $Q=0.033$ for $f_{18}$ with McDonald et al's parameters. These values are at the boarders of the range for radial modes. We conclude that neither period ratios, nor pulsation constants gave wellestablished mode identification for the frequencies of 38 Eri.

\subsection{Multi-colour photometry}

Dziembowski (1977) derived that the amplitude and phase values in different passbands can distinguish the frequencies with different horizontal quantum number $(l)$. Watson (1988) published the discriminative boxes in Johnson $B$ and $V$ colours, exactly for $A_{B-V} / A_{V}$ versus $\phi_{B-V}-$ $\phi_{V}$. Although the theoretical location of the frequencies with $l=1$ and $l=2$ partly overlap, this method was widely used in the past for mode identification. Garrido, Garcia-Lobo \& Rodriguez (1990), based on linear approximation, published the modal discrimination in pulsating stars by using Strömgren photometry. The theoretically expected location of the frequencies for $l=0,1$ and 2 were mostly overlaping for different filter combinations and the calculations were restricted to the radial pulsation constant $(Q=0.033)$. Only the $A_{b-y} / A_{y}$ amplitude ratio versus $\phi_{b-y}-\phi_{y}$ proved to be discriminative enough to compare it to the observed values. In this approach the $\phi_{b-y}-\phi_{y}$ phase difference is positive, while the other modes show negative phase differences. The later investigations using non-adiabatic pulsational treatment of the atmoshpere (Moya, Garrido \& Dupret 2004; Daszynska-Daszkiewicz, Dziembowski \& Pamyatnykh 2003) revealed that the results are highly sensitive to the convection, i.e. to the mixing length parameter $(\alpha)$. Even negative phase differences were resulted for the higher overtones $(Q=0.017)$ of the $l=0$ modes. Nevertheless, the phase difference values for frequencies with higher $l$ are always displayed from right to left, disregarding the exact values. Different approaches are followed in practice. According to Balona \& Evers (1999), the best approach to make identification is to make use of all available information. A $\chi^{2}$ goodness-fit criteria between observed amplitudes and amplitudes predicted for a given $l$ over as many wavebands as desired were used for mode identification. The method avoids the use of numerous two-colour diagrams. In one of the latest example (Breger et al. 2017), instead of the color index value $\left(\phi_{v-y}-\phi_{y}\right)$ the colour phase differences $\left(A_{v} / A_{y}\right.$ versus $\left.\phi_{v}-\phi_{y}\right)$ were used for mode identification. The amplitudes and phases are more precisely determined 
from the colour light curves of highest amplitudes than from the colour index curves. The aforementioned examples show the difficulty of the mode identification.

We tried to use what we have for the mode identification in 38 Eri. We have amplitudes and phases in Johnson $B$ and $V$ and Strömgren $b, v, y$ colours obtained in 1998. We have precise frequencies obtained from the MOST data from 2011. Although the frequencies used to be determined from the data set that is used for mode identification, even the multi-site ground-based campaign was not adequate enough to get the same well-determined frequency solution for the colours. At the same time the MOST observations were not obtained in different filters. We used a hybrid solution. We determined the color amplitudes and phases (relative to the $\mathrm{HJD}=2541113.0$ epoch) using the MOST frequencies (see in Table 7). In some cases too high amplitudes or too large phase values were obtained and thus omitted. Nevertheless, we found reasonable amplitudes and phases for 11 MOST frequencies. The amplitude ratios and phase differences are given in Table 10 for Strömgren colours $b$ and $v$, and $y$ and Johnson $B$ and $V$. Although we calculated the amplitude ratios and phase differences for the $b-y, v-y$ and $B-V$ colour indices, too, but due to the much larger error connected to the much lower amplitude and the additional mathematical calculation, the solutions were not as conclusive as for the colours. We followed Breger et al. (2017) in using the colour values instead of the color index values. Unfortunately, we do not have such a previleged situation as Breger et al. (2017) had for $4 \mathrm{CVn}$. They calculated the amplitude and phase values from season to season and presented the scatter as an error. We had to find an hybrid solution, too, for the error calculation in the case of 38 Eri. At first we tried the Monte Carlo method for error estimation, however, it revealed unrealistic errors, changing from 5-20 degrees for the phase differences and 0.07-0.3 for the amplitude ratios. This was due to the application of MOST frequencies for the colour data. However, we can use the advantage of having observations in different passbands. Different combination of the two-colour diagrams are shown in the different panels of Figure 10 displaying similar arrangement of the frequencies. The first panel showing $A_{b} / A_{y}$ versus $\phi_{b}-\phi_{y}$ is the most conclusive. There are three well-separated groups, although the theoretical investigations do not predict such a nice separation for $l=0,1$ and 2 modes (Garrido et al. 1990; Moya et al. 2004). The number beside the dots gives the ID of frequencies from Table 7. Although in the other panels the groups are not as clearly separated, the numbers help to follow how the location of a certain frequency changes from panel to panel.

A more reliable error estimation would be to average the phases of the three different colour relations given in Table 10 and use the scatter as the error. Although the theory suggests different phase differences for the different colour relations, but we can get an upper limit for the error. The averages and the scatters as errors are presented also in Table 10 and are displayed in the fourth panel of Figure 10. As the amplitude ratios are remarkably different in the colours, we used the $A_{b} / A_{y}$ amplitude ratios only for the presentation as a hybrid solution, without any additional meaning.

Supposing that the multi-colour photomery with the MOST frequencies gives reasonable approximation for the amplitude ratios and phase differences, we used them for
Table 13. Comparison of the identification with different methods: rotational splitting, sequence search (SSA) and multi-colour photometry. For multi-colour photomery identifications on different colour combinations and on the averaged phase differences are given.

\begin{tabular}{rrrcccccc}
\hline & & Rot. sp. & SSA & \multicolumn{5}{c}{ Multi-colour phot. } \\
& & & & $b, y$ & $v, y$ & $B, V$ & Average \\
ID & Freq. & $l$ & No & $l$ & $l$ & $l$ & $l$ \\
& $\left(\mathrm{~d}^{-1}\right)$ & & & & & & \\
\hline$f_{1}$ & 13.360 & 0 & II & 1 & 1 & 1 & 1 \\
$f_{2}$ & 10.476 & $1 / 2$ & I & 1 & 0 & 1 & 1 \\
$f_{3}$ & 6.477 & $2 / 3$ & III & 1 & 1 & 1 & 1 \\
$f_{4}$ & 12.228 & & I & 2 & 2 & & 3 \\
$f_{5}$ & 11.614 & & II & 2 & 2 & - & 2 \\
$f_{6}$ & 6.916 & 2 & I & 1 & 0 & - & 1 \\
$f_{7}$ & 6.070 & 1 & IV & - & - & - & - \\
$f_{8}$ & 9.199 & & IV & 2 & 1 & 2 & 2 \\
$f_{9}$ & 7.033 & 3 & - & - & - & - & - \\
$f_{11}$ & 5.498 & 3 & I & 0 & 0 & 1 & - \\
$f_{12}$ & 7.731 & 2 & II & - & - & - & - \\
$f_{13}$ & 9.604 & 2 & II & 2 & 2 & - & 3 \\
$f_{14}$ & 10.817 & & IV & - & - & - & - \\
$f_{15}$ & 5.813 & 1 & II & 0 & 0 & 0 & 0 \\
$f_{16}$ & 13.966 & & I & - & - & - & - \\
$f_{17}$ & 11.827 & & III & - & - & - & - \\
$f_{18}$ & 10.204 & 1 & III & 0 & 0 & 0 & 0 \\
\hline
\end{tabular}

mode identification given in the last four columns of Table 13. As the location of the modes with different $l$ are contradictory according to the theoretical investigations using different assumptions, we used only the general trend that is common in each investigation. The phase differences change from right to left for the $l=0,1,2$ and 3 modes. We accepted the identification for $f_{1}, f_{2}, f_{3}$ and $f_{6}$ as $l=1$ modes, for $f_{5}$, and $f_{8}$, as $l=2$ and $f_{4}$ and $f_{13}$ as $l=3$ and $f_{15}$ and $f_{18}$ as $l=0$ radial modes. We do not accept $f_{11}$ as an $l=0$ mode due to the large error bar. Although $f_{15}$ and $f_{18}$ are in the positive region the frequency ratio do not fit the regions given by Stellingwerf (1979) for the radial modes.

For comparison the third column gives the mode identification according to the rotational splitting. There is no agreement between the identification by the rotational splitting and the finally accepted identification of the multicolour photometry. The fourth column gives the numbering of sequences according to the sequence search method. Each sequence contains frequencies with all $l$ values. Taking the fast rotation of 38 Eri into account, the deviation of the real sequences from the sequences of the pure eigenmodes (Paparó et al. 2016a) is also not surprising.

Mode identification using FAMIAS (Zima 2008b,a) were carried out for comparing the theoretical values to our observational values. Using $T_{\text {eff }}=7100 \pm 120 \mathrm{~K}, \log g=$ $3.6 \pm 0.06$ and the standard assumptions for $\delta$ Scuti stars (Kurucz model, no overshooting, solar metallicity and the model of Montalban \& Dupret 2007), the best agreements are $l=1$ for $f_{1}$ and $l=2$ for $f_{5}$ and $f_{8}$ which agree with results of the identification of our hybrid method. Definitely modelling by a code for fast rotating $\delta$ Scuti stars (e.g. Lignières \& Georgeot 2009; Reese et al. 2017) would be more appropriate for 38 Eri. 


\section{SUMMARY}

The present investigation of the $\delta$ Scuti star 38 Eri resulted in a significant improvement in the description of the pulsational behaviour. We resolved the different causes of the alias discrepancies presented in previous investigations. We determined the frequency content at two epochs separated by 13 years, suggesting frequencies changing amplitude for the second epoch. Neither frequencies at 5.2 and $6.2 \mathrm{~d}^{-1}$, obtained in the colour data, appeared in the MOST data, but frequencies at $6.477 \mathrm{~d}^{-1}\left(f_{3}\right)$ and $5.498 \mathrm{~d}^{-1}\left(f_{11}\right)$ were found. Although both $f_{2}=10.476$ and $f_{18}=10.204 \mathrm{~d}^{-1}$ appeared in the MOST data, however, a frequency at $10.214 \mathrm{~d}^{-1}$ had the second highest in Johnson $B$ and $V$ colours. It is not known whether these frequencies are different members of the same rotational split triplets showing different amplitude at different epoch or not.

We found only amplitude variability and only for a single frequency, $f_{3}=6.477 \mathrm{~d}^{-1}$. We excluded beating of close pairs and resonant mode coupling as possible cause of the amplitude variation. Following Bowman et al. (2016) we concluded that variable driving/damping could be responsible for the amplitude variation.

We applied two methods for finding spacings: the rotational splitting method for the small spacings and the sequence search method for the larger spacings. We proved by modelling that the asymptotic relation for getting the rotational splittings can be used for 38 Eri. Using the scaling relation we calculated the mean density $\bar{\rho}=[0.0394,0.0554] \mathrm{gcm}^{-3}$ and surface gravity $\log g=$ $[3.40,3.66]$ cgs from the spacing obtained in the sequence search method. At the first time we compared the observables which can be obtained from these methods. The two methods seem to be numerically connected. The ratios of some shifts between the sequences and the rotational splitting found for $l=1,2$ and 3 are resulted in integer values $(2,3,5)$. We presented similar ratios for the $\delta$ Scuti star, CoRoT 102749568 as a support for our present result.

We emphashized the difficulty of the mode identification for $\delta$ Scuti stars, but we followed a positive attitude for finding some solution based on our large efforts of the ground-based multi-site multi-colour photometry and the MOST observations. We used a hybrid method for mode identification in two senses. Partly we combined the precise frequencies obtained on MOST data in 2011 and the extended ground-based multi-colour photometry from 1998. The acceptable results for the phase difference and period ratios show the capability of the hybrid method. Obtaining additional multi-colour photometry for the frequencies of high precision for the tremendous number of CoRoT and Kepler $\delta$ Scuti stars, we could provide a great step towards the identification of modes in the non-asymptotic regime of the pulsation. Secondly, we used an unusual way of getting the final mode identification based on the multi-colour photometry. We present the discriminative panels of the different colour combinations separately, however, we stepped further. We calculated the averaged phase differences of the different color combinations and used the scatter of the independent values to the average as an error. We used for mode identification the general trends of the theoretical calculations instead of certain boxes derived. The phase differences change from right to left for the $l=0,1,2$, and 3 modes. This method resulted in two modes with $l=0\left(f_{15}\right.$ and $\left.f_{18}\right)$. A third frequency $\left(f_{11}\right)$ resulted also in a positive phase difference, but with a large error bar. Four modes $\left(f_{1}, f_{2}, f_{3}\right.$ and $f_{6}$ ) located in the low negative region are identified as $l=1$ modes. Two well-separated higher phase difference values appear to suggest an identification with $l=2$ for $f_{5}$ and $f_{8}$, and with $l=3$ for $f_{13}$ and $f_{4}$. FAMIAS resulted in the same identification for $f_{1}, f_{5}$ and $f_{8}$.

There is no agreement between the identification based on the rotational splitting and the multi-colour photometry. The sequences do not contain modes with the same $l$ values. At this moment we may not conclude that we can have mode identification for $\delta$ Scuti stars based only on the frequencies. However, 38 Eri is only one example. We have precise frequencies for plenty of $\delta$ Scuti stars by the CoRoT and Kepler space missions and we will have even more by the forthcoming TESS and PLATO missions. We believe that new approaches are needed for getting the information that are at our hands in the space data. Maybe, the frequencies of even such a complex $\delta$ Scuti star as 38 Eri could be successfully identified in space data with longer time base, especially if the theoretical mode identification is also applied (Reese et al. 2017).

\section{ACKNOWLEDGEMENTS}

MP was supported by Soros Foundation. She also thanks the staff of SAAO. MP thanks Gerald Handler for his remarks and comments on behalf of the late Robert Shobbrook. ZK observed in the frame of the exchange agreement between the Hungarian Academy of Sciences and CONACYT.This work was supported by the ESA PECS Grant No 4000103541/11/NL/KML. ÁS was supported by the János Bolyai Research Scholarship of the Hungarian Academy of Sciences, and he also acknowledges the financial support of the Hungarian NKFIH Grant K-113117. Spectroscopic observations made with the Mercator Telescope, operated on the island of La Palma by the Flemmish Community, at the Spanish Observatorio del Roque de los Muchachos of the Instituto de Astrofísica de Canarias. ÁS, JMB and ZsB acknowledge the financial support of the Hungarian NKFIH Grants K-115709 and K-119517. ZsB acknowledges the support provided from the National Research, Development and Innovation Fund of Hungary, financed under the PD_17 funding scheme, project No. PD123910. AK acknowledges the Science and Education Ministry of Kazakhstan (grant No. 0075/GF4). AFJM is grateful for financial aid from NSERC (Canada) and FQRNT (Quebec). AGH acknowledges funding support from Spanish public funds for research under project ESP2015-65712C5-5-R (MINECO/FEDER), and from project RYC-201209913 under the 'Ramón y Cajal' programme of the Spanish MINECO. We acknowledge the International Space Science Institute (ISSI) for supporting the SoFAR international team. ${ }^{2}$ We thank to the anonymous referee for the very thorough work.

2 http://www.issi.unibe.ch/teams/sofar/ 


\section{REFERENCES}

Aerts, C. et al., 2006, ApJ, 642, L165

Baglin, A. Breger, M., Chevalier, C., Hauck, B., Le Contel, J. M., Sareyan, J. P., Valtier, J. C., 1973, A\&A, 23, 221

Baglin, A. et al., 2006, in Fridlund, M., Baglin, A., Lochard, J., Conroy, L., eds, ESA SP 1036, The CoRoT Mission, Prelanch Status, Stellar Seismology and Planet Finding, ESA Publications Division, Noordwijk, p. 33

Balona, L., 2000, MNRAS, 318, 289

Balona, L., Evers, E. A., 1999, MNRAS, 302, 349

Balona, L., Baran, A. S, Daszynska-Daszkiewicz, J., de Cat, P., 2015, MNRAS, 451, 1445

Barceló Forteza, S., Michel, E., Roca Cortés, T., García, R. A., 2015, ApJ, 579, 133

Bowman, D. M., Kurtz, D. W., Breger, M., Murphy, S. J., Holdsworth, D. L., 2016, MNRAS, 460, 1970

Breger, M., 1979, PASP, 100, 751

Breger, M., 2000a, MNRAS, 313, 129

Breger, M., 2000b, in Breger, M., Montgomery, M. H. eds., ASP Conf. Ser. 210, Delta Scuti and Related Stars, Astron. Soc. Pacific, San Francisco, p. 3.

Breger, M., 2009, in Guzik, J. A., Bradley, P. A., eds, AIP Conf Ser. Vol 1170, Stellar Pulsation: Challenges for theory and observations. AIP, Melville, New York, p. 410

Breger, M., 2010, in Sterken, C., Samus, N., Szabados, L., eds, Variable Stars, the Galactic halo and Galaxy Formation, Sternberg Astronomical Institute of Moscow Univ., Russia, p. 95

Breger, M., Bregman, J. N., 1975, ApJ, 200, 343

Breger, M., Bischof, K. M., 2002, A\&A, 385, 537

Breger, M., Pamyatnykh, A. A., 2006, Mem. Soc. Astron. Italiana, 77. 295

Breger, M., Montgomery, M. H., 2014, ApJ, 783, id.89

Breger, M., Pamyatnykh, A. A., Pikall, H., Garrido, R., 1999, A\&A, 341, 151

Breger, M., Lenz, P., Pamyatnykh, A. A., 2009, MNRAS, 396, 291

Breger, M., Montgomery, M. H., Lenz, P., Pamyatnykh, A. A., 2017, A\&A, 599, A116

Brickhill, A. J., 1975, MNRAS, 170, 405

Borucki, W. J. et al., 2010, Science, 327, 977

Chadid, M., et al., 2010, A\&A, 510, A39

Chen, X. H., Li, Y., 2017, ApJ, 838, id.31

Chen, X. H., Li, Y., Lai, X. J., Wu, T., 2016, A\&A, 593, A69

Chen, X. H., Li, Y., Lin, G. F., Chen, Y. H., Guo, J. J., 2017, ApJ, 834, id.146

Daszynska-Daszkiewicz, J., Dziembowski, W. A., Pamyatnykh, A. A., 2003, A\&A, 407, 999

Dziembowski, W., 1977, Acta Astron., 27, 203

Dziembowski, W. A., Goode, P., 1992, ApJ, 394, 670

Fitch, W. S., 1981, ApJ, 249, 218

Garrido, R., Garcia-Lobo, E., Rodriguez, E., 1990, A\&A, 234, 262

García Hernández, A., et al., 2009, A\&A, 506, 79

García Hernández, A., et al., 2013, A\&A, 559, A63

García Hernández, A., Martín-Ruiz, S., Monteiro, M. J. P. F. G., Suárez, J. C., Reese, D. R., Pascual-Granado, J., Garrido, R., 2015, ApJ, 811, id.29

García Hernández, A., et al, 2017, MNRAS, 471, 140

Gizon, L., Solanki, S. K., 2003, ApJ, 589, 1009

Gray, R. O., et al, 2006, AJ, 132, 161

Guzik, J. A., Kosak, K., Bradley, P. A., Jackievicz, J., 2016, in Benvenuti, P. ed., IAU Focus Meetings, Vol. 29B, Astronomy in Focus as presented at the IAU XXIX General Assembly, Cambridge Univ. Press, p. 560

Handler, G., et al, 1997, MNRAS, 286, 303

Jørgensen, H. E., Johansen, K. T., Olsen, E. H., 1971, A\&A, 12, 223
Jørgensen, H. E., Nørgaard-Nielsen, H. U., 1975, A\&A, 19, 235

Hermes, J. J., Kepler, S. O., Montgomery, M. H., Giannias, M., Castanheira, B. G., Winget, D. E., 2015, in Dufour, P., Bergeron, P., Fontaine, G. eds., ASP Conf. Ser. 493, 19th European Workshop on White Dwarfs, Astronomical Society of the Pacific, San Francisco, p. 53

Kilkenny, D., Balona, L. A., Carter, D. B., Ellis, D. T., Woodhouse, G. F. W. 1988, MNASSA, 47, 69

Koch, D. G. et al., 2010, ApJ, 713, L79

Kolláth, Z., 1990, Occ. Tech. Notes Konkoly Obs., No. 1.

Ledoux, P., 1951, ApJ, 114, 373

Lenz, P., Breger, M., 2005, CoAst. 146, 53

Lignières, F., Georgeot, B., 2008, Phys. Rev. E, 78, 6215

Lignières, F., Georgeot, B., 2009, A\&A, 500, 1173

Lignières, F., Rieutord, M., Reese, D. R., 2006, A\&A, 455, 607

Lignières, F., Rieutord, M., Ballot, J., 2010, Astron. Nachr., 331, 1053

Matthews, J. M., 2004, in Maeder, A., Eenens, Ph., eds, IAU Symp. 215, Stellar Rotation, Astron. Soc. of the Pac., San Francisco, p. 189

Matthews, J. M., Kuschning, R., Guenther, D. B., Walker, G. A. H., Moffat, A. F. J., Rucinski, S. M., Sasselov, D., Weiss, W. W., 2004, Nature, 430, 51

McDonald, I., Zijlstra, A. A., Watson, R. A., 2017, MNRAS, 471, 770

Medupe, R., Kurtz, D. W., Elkin, V. G., Mguda, Z., Mathys, G., 2015, MNRAS, 446, 1347

Michel, E., et al., 2017, in Monteiro, M. J. P. F. G., Cunha, M. S., Ferreira, J. M. T. S. eds., EPJ Web of Conf., 160, Seismology of the Sun and the Distant Stars - Using Today's Successes to Prepare the Future, id.03001

Montalban, J., Dupret, M.-A., 2007, A\&A, 470, 991

Moya, A., Garrido, R., Dupret, M.-A., 2004, A\&A, 414, 1081

Moya, A., Suárez, J. C., García Hernández, A., Mendoza, M. A., 2017, MNRAS, 471, 2491

Osaki, J., 1975, PASJ, 27, 237

Paparó, M. et al., 2013, A\&A, 557, A27

Paparó, M., Benkő, J. M., Hareter, M., Guzik, J. A., 2016a, ApJ, 822 , id. 100

Paparó, M., Benkő, J. M., Hareter, M., Guzik, J. A., 2016b, ApJS, 224 , id. 41

Pascual-Granado, J., Garrido, R., Suárez, J. C., 2015, A\&A, 575, A78

Paxton, B, Bildsten, L., Dotter, A., Herwig, F., Lesaffre, P., Timmes, F., 2011, ApJS, 192, 3

Paxton, B., et al., 2013, ApJS, 208, 4

Paxton, B., et al., 2015, ApJS, 220, 15

Poretti, E., 1989, A\&A, 220, 144

Poretti, E., Mantegazza, L., Antonello, E., 1987, A\&A, 181, 273

Poretti, E., Antonello, E., Mantegazza, L., 1988, A\&A, 199, 191

Raskin, G. et al., 2011, A\&A, 526, A69

Reese, D. R., Lignières, F., Rieutord, M., 2008, A\&A, 481, 449

Reese, D. R., Thompson, M. J., MacGregor, K. B., Jackson, S., Skumanich, A., Metcalfe, T. S., 2009, A\&A, 506, 183

Reese, D. R., Lignières, F., Ballot, J., Dupret, M.-A., Barban, C., van't Veer-Menneret, C., MacGregor, K. B., 2017, A\&A, 601, A130

Roxburgh, I. W., 2006, A\&A, 454, 883

Soufi, F., Goupil, M.-J., Dziembowski, W. A., 1998, A\&A, 334, 911

Stellingwerf, R. F., 1979, ApJ, 227, 935

Suárez, J. C., Garrido, R., 2006, Mem. Soc. Astron. Italiana, 77, 502

Suárez, J. C., Garrido, R., Goupil, M. J., 2006, A\&A, 447, 649

Suárez, J. C., Garrido, R., Moya, A., 2007, A\&A, 474, 961

Suárez, J. C., García Hernández, A., Moya, A., Rodrigo, C., Solano, E., Garrido, R., Rodón, J. R., 2014, A\&A, 563, A7 van Leeuwen, F., 2007, A\&A, 474, 653 
Vorontsov, S. V., 1981, Soviet Ast., 25, 724

Vorontsov, S. V., 1983, Sol. Phys., 82, 379

Walker, G. A. H. et al., 2003, PASP, 115, 1023

Walker, G. A. H. et al., 2005, ApJ, 635, L77

Watson, R. D., 1988, Ap\&SS, 140, 255

Winget, D. E. et al., 1991, ApJ, 378, 326

Yang, S., Walker, G. A. H., 1986, PASP, 98, 1156

Zima, W., 2008a, CoAst, 155, 17

Zima, W., 2008b, CoAst, 157, 387

\section{Affiliations}

${ }^{1}$ Konkoly Observatory, MTA CSFK, Konkoly Thege M. u. 15-17., H-1121 Budapest, Hungary

${ }^{2}$ Eötvös Loránd University, Savaria Department of Physics, Károlyi G. tér 4., H-9700 Szombathely, Hungary

${ }^{3}$ Australian National University, Siding Spring Observatory, Coonabarabran, NSW 2137, Australia

${ }^{4}$ Department of Physics and Astronomy, University of British Columbia, 6224 Agricultural Road, Vancouver, BC V6T 1Z1, Canada

${ }^{5}$ Stellar Astrophysics Centre, Department of Physics and Astronomy, Aarhus University. 120 Ny Munkegade, DK-8000 Aarhus C, Denmark

${ }^{6}$ Korea Astronomy and Space Science Institute, 39-18 Hwaamdong, Yuseong-gu, Daejeon 34055, Republic of Korea

${ }^{7}$ Avcorp Industries, Inc. 10025 River Way, Delta, BC, V4G 1M7, Canada

${ }^{8}$ Department of Physics, Alzahra University, P.O. Box 1993893973, Tehran, Iran

${ }^{9} 8100$ Barstow St. NE, Apt 6104, Albuquerque, NM, 87122, USA

${ }^{10}$ Fesenkov Astrophysical Institute, Observatory 23, 050020, Almaty, Kazakhstan

${ }^{11}$ Department of Theoretical Physics and Cosmology, University of Granada (UGR), E-18071 Granada, Spain

${ }^{12}$ Instituto de Astronomía, UNAM, Apartado Postal 70-264, Ciudad México, CDMX, C. P. 04510, Mexico

${ }^{13}$ Institut für Astronomie, Universität Wien, Türkenschanzstrasse 17, A-1180 Wien, Austria

${ }^{14}$ Institut für Kommunikationsnetze und Satellitenkommunikation, Technische Universität Graz, Infeldgasse 12, 8010 Graz, Austria

${ }^{15}$ Département de physique and Centre de Recherche en Astrophysique du Québec (CRAQ), Université de Montréal, C.P. 6128, Succ. Centre-Ville, Montréal, QC H3C 3J\%, Canada

${ }^{16}$ Canadian Coast Guard College, Dept. of Arts, Sciences, and Languages, Sydney, Nova Scotia, B1R 2J6, Canada

${ }^{17}$ Department of Astronomy and Astrophysics, University of Toronto, Toronto, ON M5S 3H4, Canada

${ }^{18}$ International Centre for Radio Astronomy Research, University of Western Australia 35 Stirling Hwy, Crawley, WA 6009, Australia

This paper has been typeset from a $\mathrm{T}_{\mathrm{E}} \mathrm{X} / \mathrm{LAT}_{\mathrm{E}} \mathrm{X}$ file prepared by the author. 